\title{
Photoactivatable Dopamine and Sulpiride to Explore the Function of Dopaminergic Neurons and Circuits
}

\author{
Naeem Asad, ${ }^{\dagger}$ Duncan E. McLain, ${ }^{\dagger, \downarrow}$ Alec F. Condon, ${ }^{\S}$ Sangram Gore, ${ }^{\dagger}$ \\ Shahienaz E. Hampton, ${ }^{\dagger}$ Sauparnika Vijay, ${ }^{\dagger}$ John T. Williams, ${ }^{*, \S}$ and Timothy M. Dore ${ }^{*, \dagger}$, \\ ${ }^{\dagger}$ New York University Abu Dhabi, Saadiyat Island, PO Box 129188, Abu Dhabi, United Arab Emirates \\ ${ }^{\ddagger}$ Department of Chemistry, University of Georgia, Athens, Georgia 30602, USA \\ $\S$ Vollum Institute, Oregon Health and Science University, Portland, Oregon, 97239, USA
}

\section{Table of Contents}

Figure S1. Instability of RuBi-DA in brain slices. .53

Scheme S1. Synthesis of MOM-CyHQ-OH and CyHQ-OMs.

Figure S2. UV-vis spectra of CyHQ-O-DA, CyHQ-N-DA, (CyHQ) ${ }_{2}-\mathrm{O}, \mathrm{O}-\mathrm{DA},(\mathrm{CyHQ})_{2}-\mathrm{N}, \mathrm{O}-\mathrm{DA}$, and CyHQ-sulpiride in KMOPS buffer.

Figure S3. Time courses for the photoreaction of CyHQ-N-DA, $(\mathrm{CyHQ})_{2}-\mathrm{O}, \mathrm{O}-\mathrm{DA}$, and $(\mathrm{CyHQ})_{2}-$ N,O-DA with 365-nm light.

Figure S4. Time courses for the photoreaction of CyHQ-N-DA, $(\mathrm{CyHQ})_{2}-\mathrm{O}, \mathrm{O}-\mathrm{DA}$, and $(\mathrm{CyHQ})_{2}-$ N,O-DA with 405-nm light.

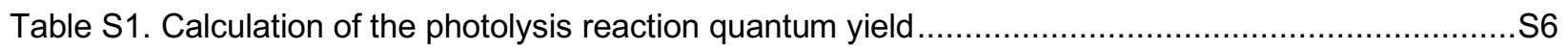

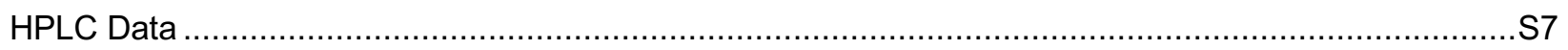

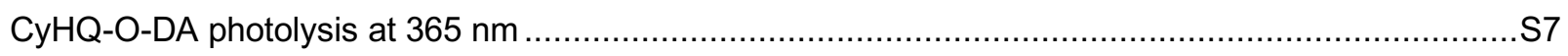

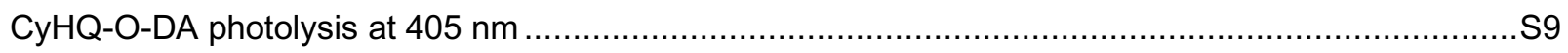

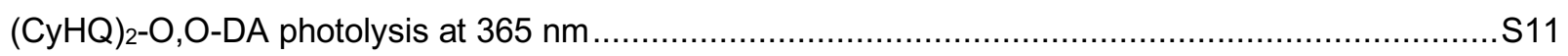

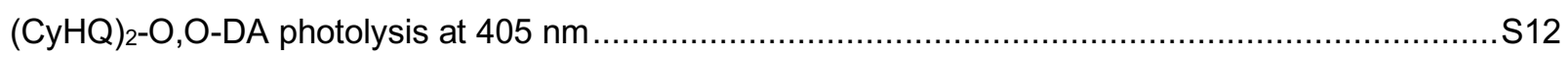

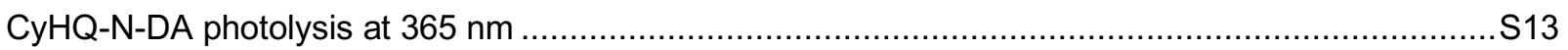

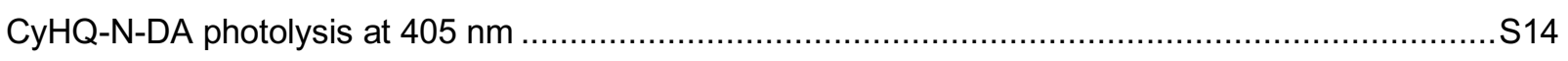

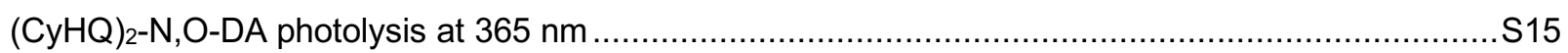

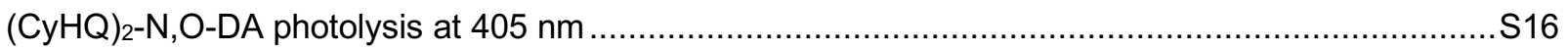

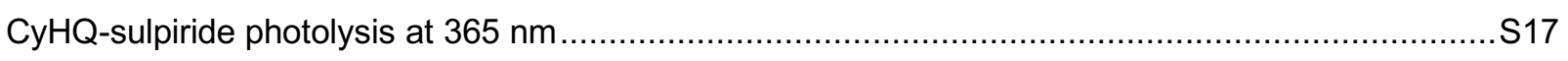

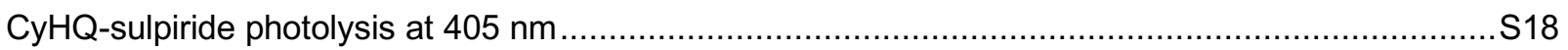

Figure S5. Time courses for the photoreaction of CyHQ-O-DA and (CyHQ) ${ }_{2}-\mathrm{O}, \mathrm{O}-\mathrm{DA}$ with 740-nm

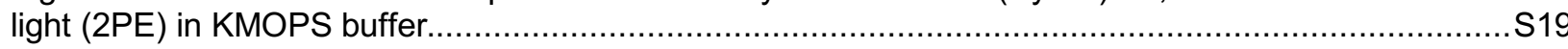

Figure S6. Time course for the photoreaction of $\mathrm{CyHQ}$-sulpiride with 740-nm light (2PE) in KMOPS buffer.

Table S2. Data for the calculation of 2-photon uncaging action cross-sections $\left(\delta_{\mathrm{u}}\right)$.............................. 20

Figure S7. The current produced by CyHQ-O-DA (1 mM) is not fully blocked by sulpiride...................S21

Figure S8. CyHQ-sulpiride alone has a small level of antagonism prior to photolysis. ........................S2 1

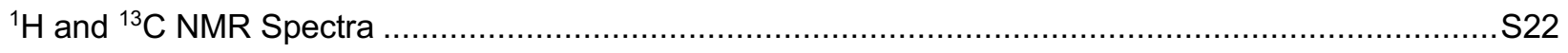

tert-Butyl (3-((8-cyano-7-(methoxymethoxy)quinolin-2-yl)methoxy)-4hydroxyphenethyl)carbamate and tert-Butyl (4-((8-cyano-7-(methoxymethoxy)quinolin-2yl)methoxy)-3-hydroxyphenethyl)carbamate (MOM-CyHQ-O-DA-Boc) 
2-(3-((8-cyano-7-hydroxyquinolin-2-yl)methoxy)-4-hydroxyphenyl)ethan-1-aminium 2,2,2trifluoroacetate and 2-(4-((8-cyano-7-hydroxyquinolin-2-yl)methoxy)-3-hydroxyphenyl)ethan-1aminium 2,2,2-trifluoroacetate (CyHQ-O-DA)

2-(3,4-bis((8-cyano-7-hydroxyquinolin-2-yl)methoxy)phenyl)ethan-1-aminium 2,2,2trifluoroacetate ((CyHQ) 2 -O,O-DA)

(8-Cyano-7-(methoxymethoxy)quinolin-2-yl)methyl (3,4-dihydroxyphenethyl)carbamate (MOMCyHQ-N-DA).

(8-Cyano-7-hydroxyquinolin-2-yl)methyl (3,4-dihydroxyphenethyl)carbamate (CyHQ-N-DA) S26

(8-Cyano-7-hydroxyquinolin-2-yl)methyl (3-((8-cyano-7-hydroxyquinolin-2-yl)methoxy)-3hydroxyphenethyl)carbamate and (8-cyano-7-hydroxyquinolin-2-yl)methyl (4-((8-cyano-7hydroxyquinolin-2-yl)methoxy)-3-hydroxyphenethyl)carbamate ((CyHQ)2-N,O-DA)

1-((8-cyano-7-hydroxyquinolin-2-yl)methyl)-1-ethyl-2-((2-methoxy-5-

sulfamoylbenzamido)methyl)pyrrolidin-1-ium (CyHQ-Sulpiride) 2,2,2-trifluoroacetate

References 


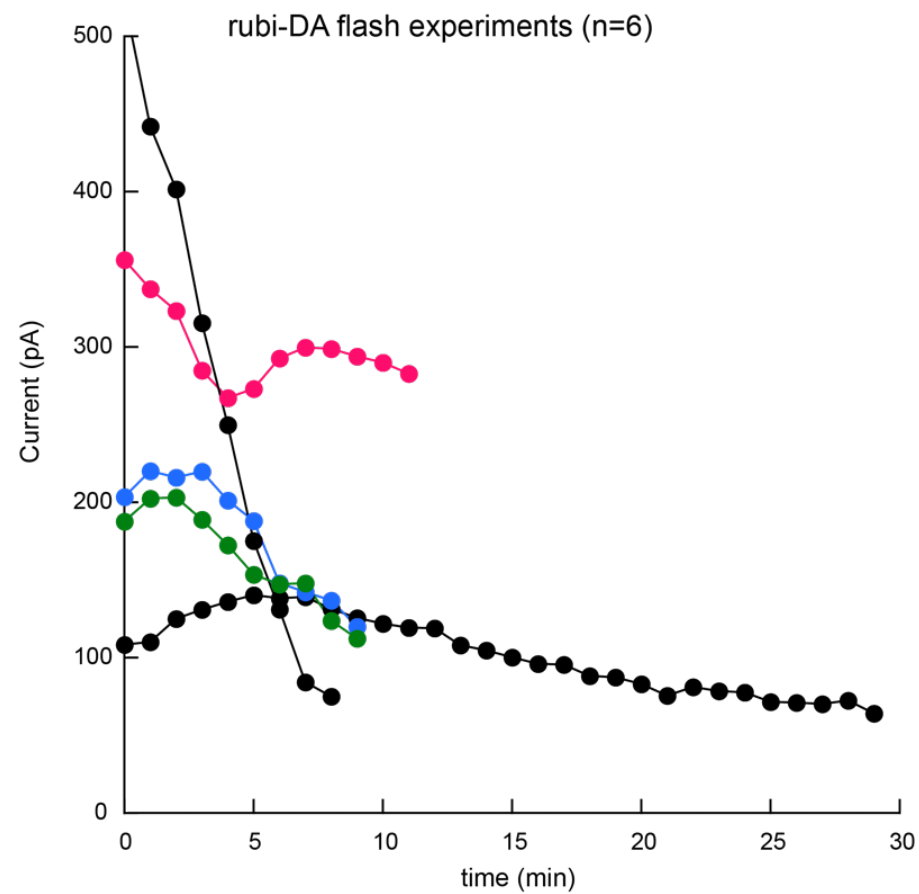

Figure S1. Instability of RuBi-DA in brain slices.

Plot of the outward GIRK current induced by photolysis of RuBi-DA ( $50 \mu \mathrm{M})$ made from recordings from dopamine neurons $(n=6$, slices) in horizontal brain slices. Flashes of 365-nm light $(5-40 \mathrm{~ms})$ were applied at 1-min intervals. In some cases, the amplitude of the current induced by dopamine was initially steady, but in all cases the amplitude declined after 5-10 min. Even when a small current was evoked using a 5-ms flash, the current declined continuously over a period of $30 \mathrm{~min}$ (black dots).

Scheme S1. Synthesis of MOM-CyHQ-OH and CyHQ-OMs.
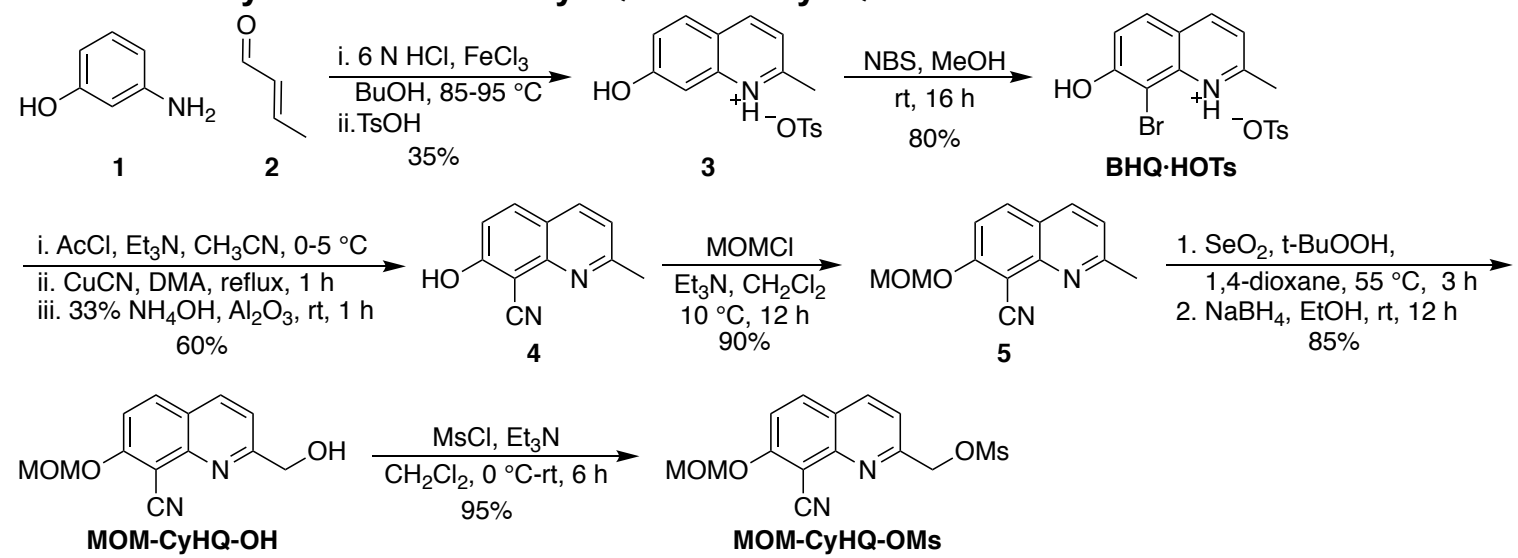


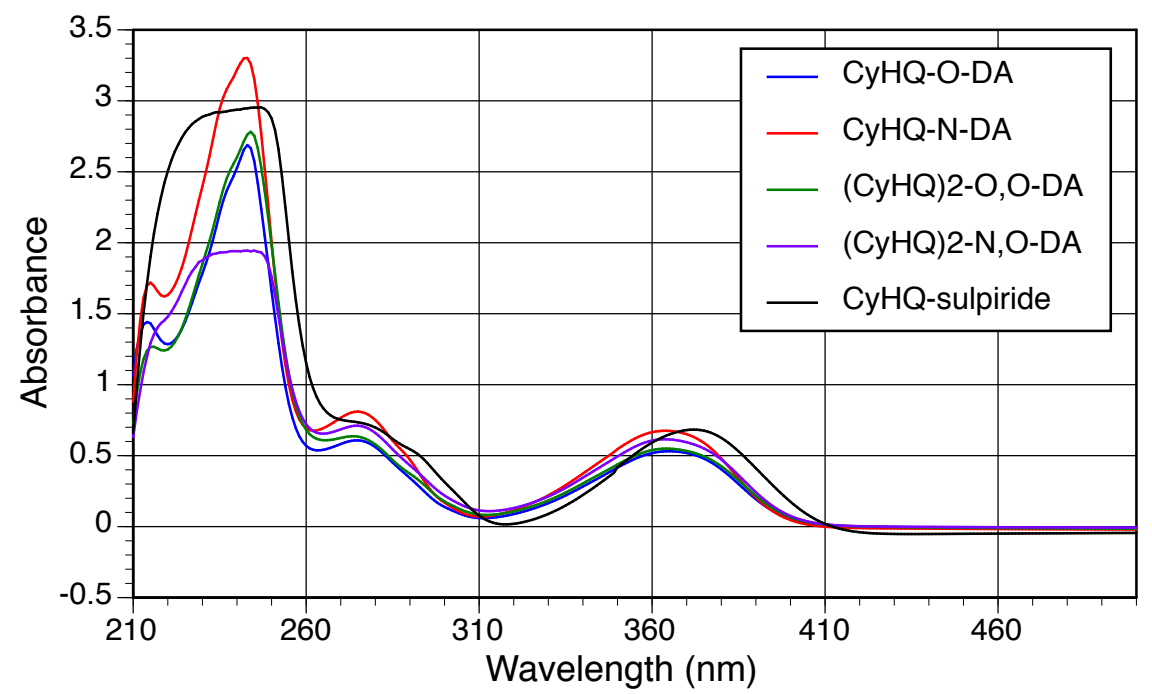

Figure S2. UV-vis spectra of CyHQ-O-DA, CyHQ-N-DA, (CyHQ) $2-O, O-D A,(C y H Q)_{2}-N, O-D A$, and CyHQ-sulpiride in KMOPS buffer. 


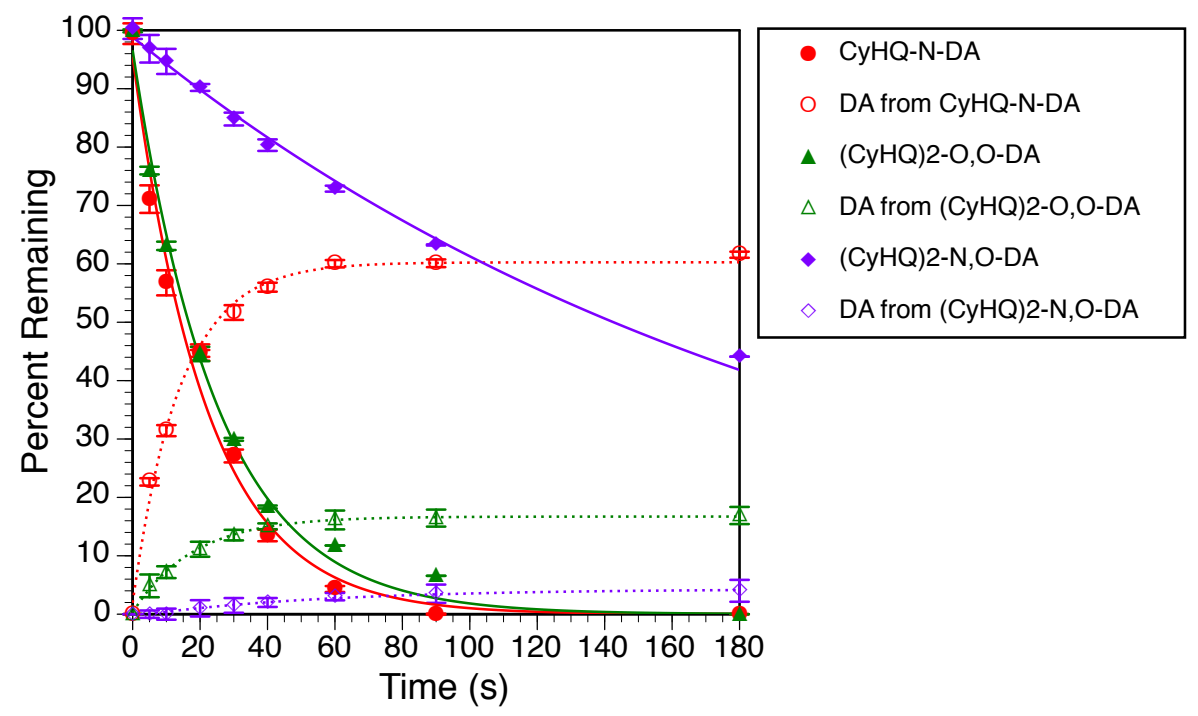

Figure S3. Time courses for the photoreaction of CyHQ-N-DA, (CyHQ) ${ }_{2}-\mathrm{O}, \mathrm{O}-\mathrm{DA}$, and (CyHQ)2-N,ODA with 365-nm light.

Solid lines are the least-squares fit to an exponential decay and dashed lines are an exponential rise to max for each measurement of dopamine product. Error bars represent the standard deviation of the average of three measurements.

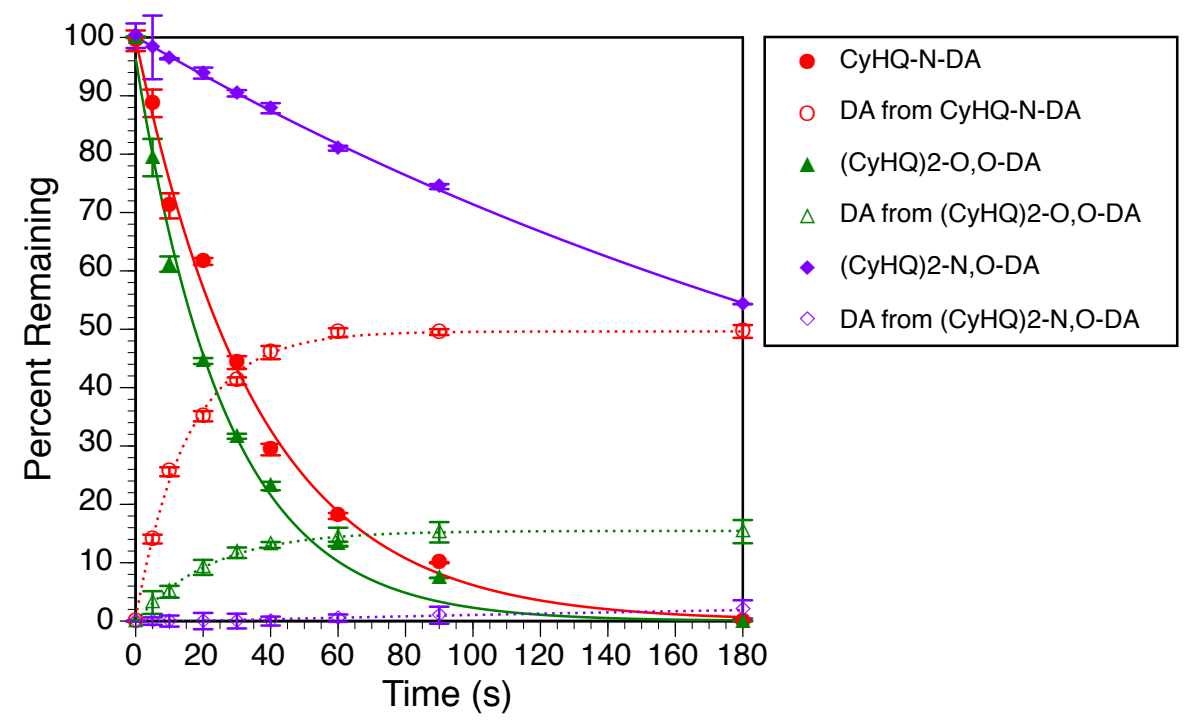

Figure S4. Time courses for the photoreaction of CyHQ-N-DA, (CyHQ) ${ }_{2}-\mathrm{O}, \mathrm{O}-\mathrm{DA}$, and (CyHQ) $2-\mathrm{N}, \mathrm{O}-$ DA with 405-nm light.

Solid lines are the least-squares fit to an exponential decay and dashed lines are an exponential rise to max for each measurement of dopamine product. Error bars represent the standard deviation of the average of three measurements. 
Table S1. Calculation of the photolysis reaction quantum yield

The quantum yield $\left(\Phi_{u}\right)$ of the photolysis reaction was calculated using the following equation: ${ }^{1-4}$

$\Phi_{u}=\left(I \sigma t_{90 \%}\right)^{-1}$

$I=$ irradiation intensity (determined by potassium ferrioxalate actinometry) ${ }^{5}$

$\sigma=$ the decadic extinction coefficient $(1000 \times \varepsilon)$ at $365 \mathrm{~nm}$ or at $405 \mathrm{~nm}$

$t_{90 \%}=$ time required to consume $90 \%$ of the starting material

\begin{tabular}{|c|c|c|c|c|c|c|}
\hline Compound & $\begin{array}{c}I \\
\left(\text { Einstein } \mathrm{cm}^{-2} \mathrm{~s}^{-1}\right) \\
365 \mathrm{~nm}\end{array}$ & $\begin{array}{c}\sigma \\
\left(\mathrm{cm}^{2} \mathrm{~mol}^{-1}\right) \\
365 \mathrm{~nm}\end{array}$ & $\begin{array}{c}t_{90 \%} \\
(\mathrm{~s}) \\
365 \mathrm{~nm}\end{array}$ & $\begin{array}{c}I \\
\left(\text { Einstein } \mathrm{cm}^{-2} \mathrm{~s}^{-1}\right) \\
405 \mathrm{~nm}\end{array}$ & $\begin{array}{c}\sigma \\
\left(\mathrm{cm}^{2} \mathrm{~mol}^{-1}\right) \\
405 \mathrm{~nm}\end{array}$ & $\begin{array}{c}t_{90 \%} \\
(s) \\
405 \mathrm{~nm}\end{array}$ \\
\hline CyHQ-O-DA & $2.16 \times 10^{-8}$ & $5.30 \times 10^{6}$ & 46 & $2.74 \times 10^{-7}$ & $2.80 \times 10^{5}$ & 65 \\
\hline$(\mathrm{CyHQ})_{2}-\mathrm{O}, \mathrm{O}-\mathrm{DA}$ & $1.75 \times 10^{-8}$ & $5.48 \times 10^{6}$ & 55 & $2.80 \times 10^{-7}$ & $3.24 \times 10^{5}$ & 58 \\
\hline CyHQ-N-DA & $1.64 \times 10^{-8}$ & $6.70 \times 10^{6}$ & 48 & $2.43 \times 10^{-7}$ & $2.51 \times 10^{5}$ & 82 \\
\hline$(\mathrm{CyHQ})_{2}-N, O-D A$ & $1.29 \times 10^{-8}$ & $8.30 \times 10^{6}$ & 466 & $1.28 \times 10^{-7}$ & $6.31 \times 10^{5}$ & 684 \\
\hline CyHQ-sulpiride & $4.47 \times 10^{-8}$ & $6.50 \times 10^{6}$ & 18 & $3.07 \times 10^{-7}$ & $8.30 \times 10^{5}$ & 18 \\
\hline
\end{tabular}




\section{HPLC Data}

Instrument: Agilent 1290 Infinity series uHPLC

Column: Zorbax Eclipse Plus C18 column

Mobile phase: $A=0.1 \%$ trifluoroacetic acid in water and $B=$ acetonitrile

Gradient: $5 \%$ B to $100 \%$ B over $10 \mathrm{~min}$, then re-equilibration to $5 \%$ B before the next run

Flow rate: $0.3 \mathrm{~mL} / \mathrm{min}$.

Observation wavelength: $280 \mathrm{~nm}$

Dopamine, sulpiride, $\mathrm{CyHQ}-\mathrm{OH}$, and $\mathrm{CyHQ}$-protected dopamines have absorptivity at this wavelength in the acidic mobile phase (although the absorptivity of $\mathrm{CyHQ}$ is quite weak at this wavelength relative to dopamine and sulpiride), enabling simultaneous observation of all species.

CyHQ-O-DA photolysis at $365 \mathrm{~nm}$

Reaction monitored at $280 \mathrm{~nm}$ for CyHQ-O-DA ( $t_{R}=4.99$ and $\left.5.18 \mathrm{~min}\right)$, dopamine ( $\left.t_{R}=0.95 \mathrm{~min}\right)$, and $\mathrm{CyHQ}-\mathrm{OH}\left(t_{\mathrm{R}}=3.1 \mathrm{~min}\right)$.

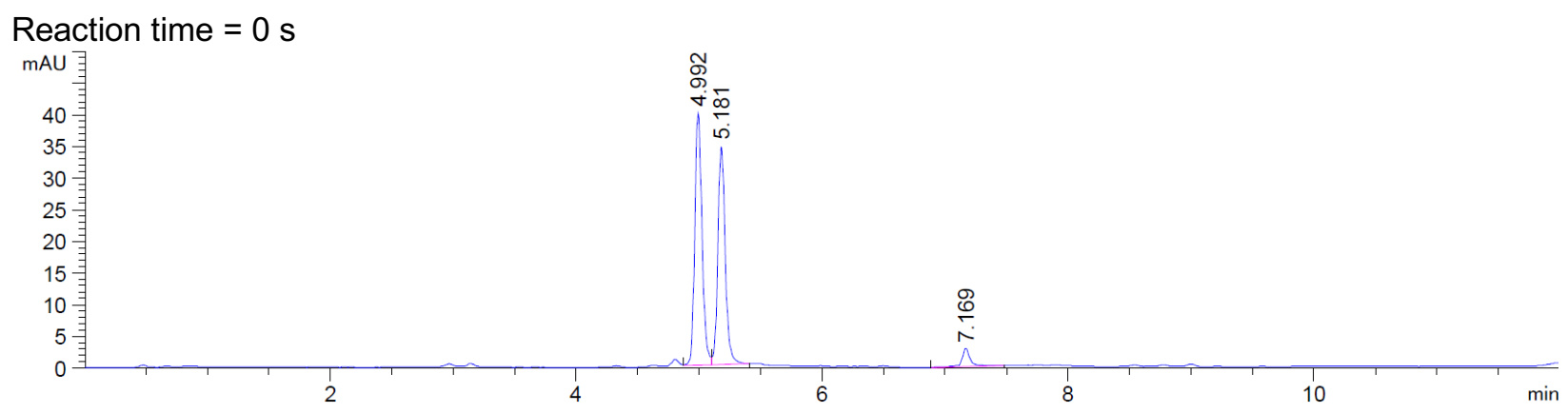

\section{Reaction time $=5 \mathrm{~s}$}

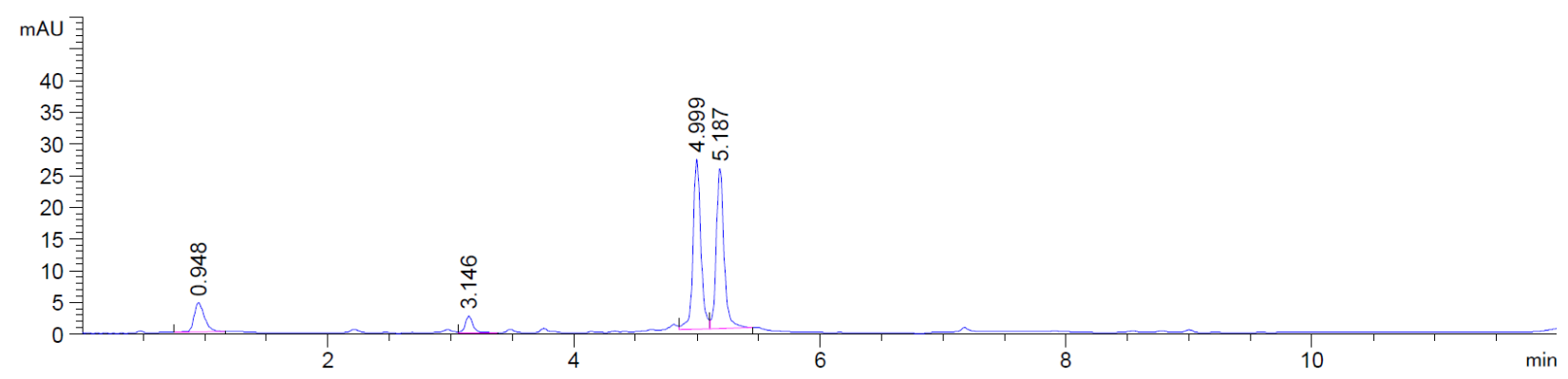

Reaction time $=30$ seconds

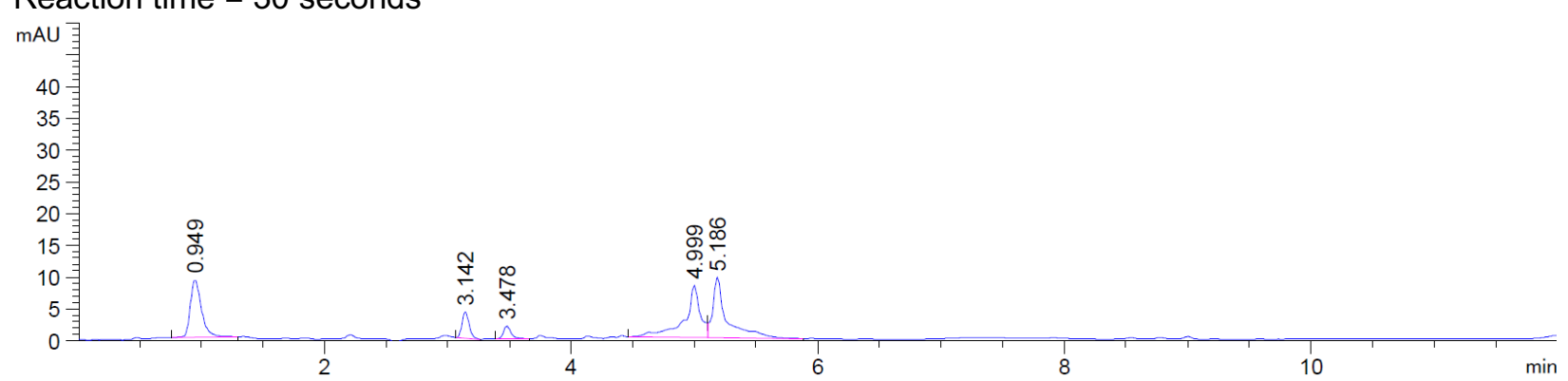



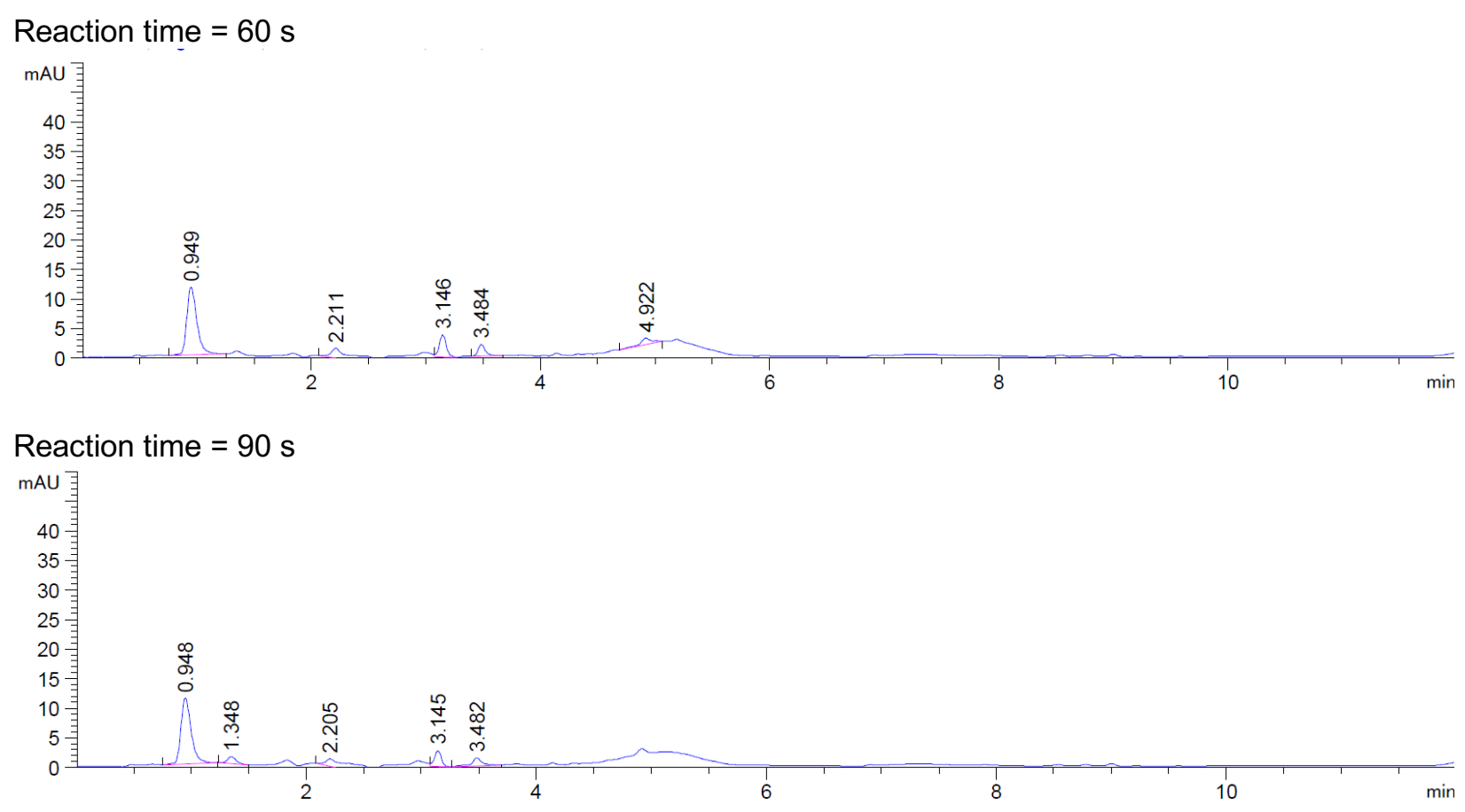
CyHQ-O-DA photolysis at $405 \mathrm{~nm}$

Reaction monitored at $280 \mathrm{~nm}$ for CyHQ-O-DA ( $t_{R}=4.99$ and $\left.5.18 \mathrm{~min}\right)$, dopamine $\left(t_{\mathrm{R}}=0.95 \mathrm{~min}\right)$, and $\mathrm{CyHQ}-\mathrm{OH}\left(t_{\mathrm{R}}=3.1 \mathrm{~min}\right)$.

Reaction time $=0 \mathrm{~s}$

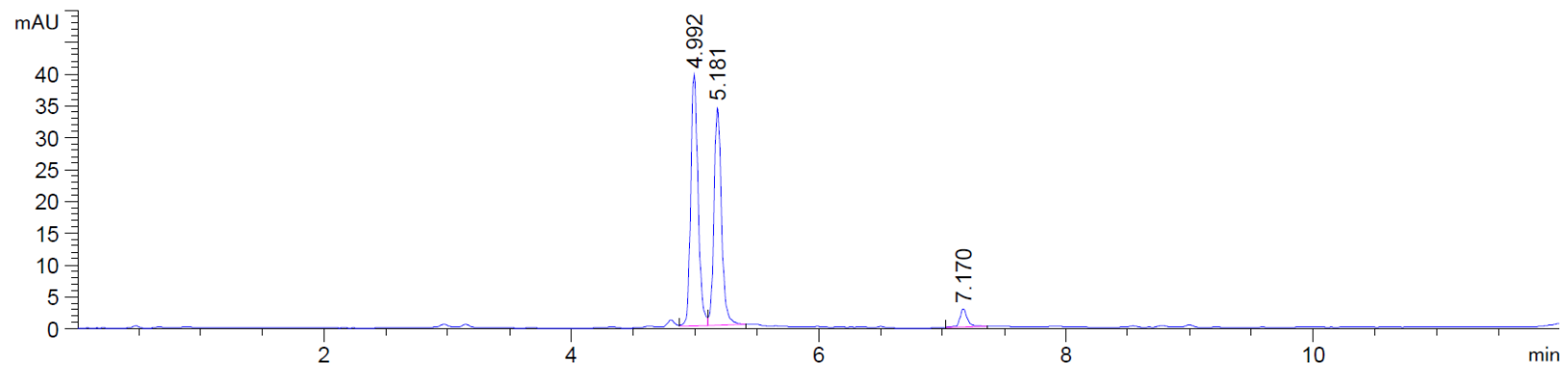

Reaction time $=5 \mathrm{~s}$

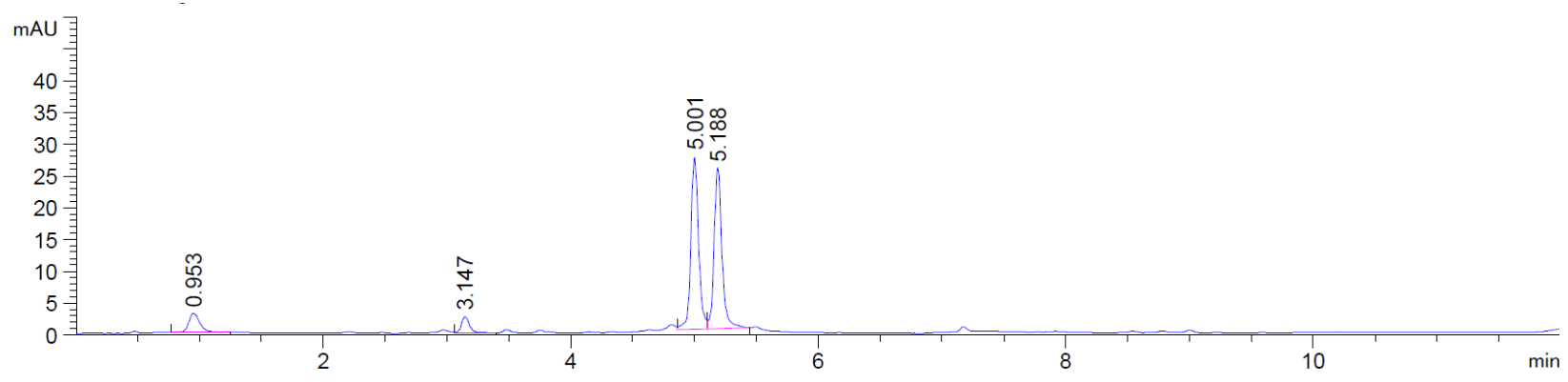

Reaction time $=40 \mathrm{~s}$

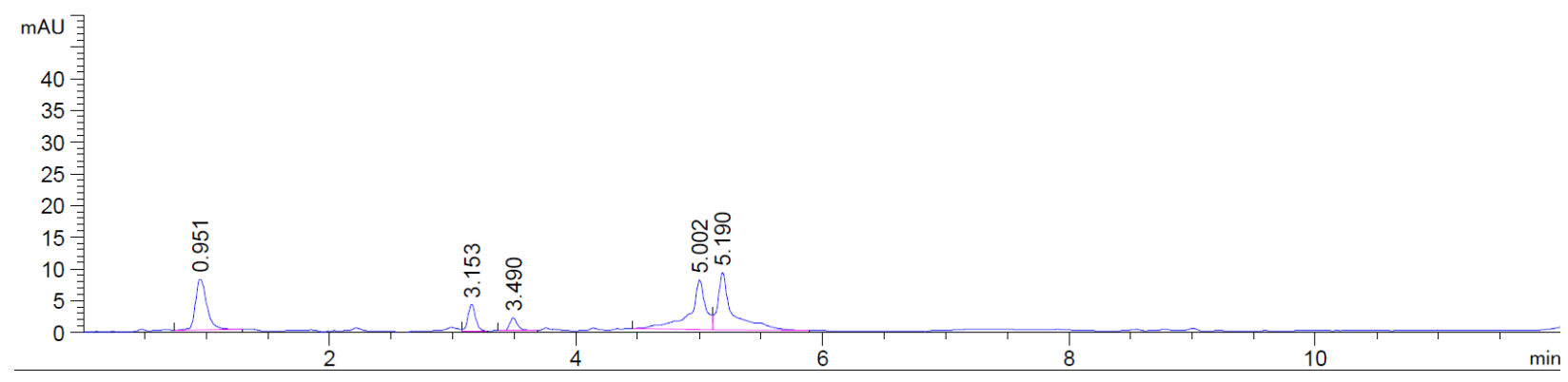

Reaction time $=60 \mathrm{~s}$

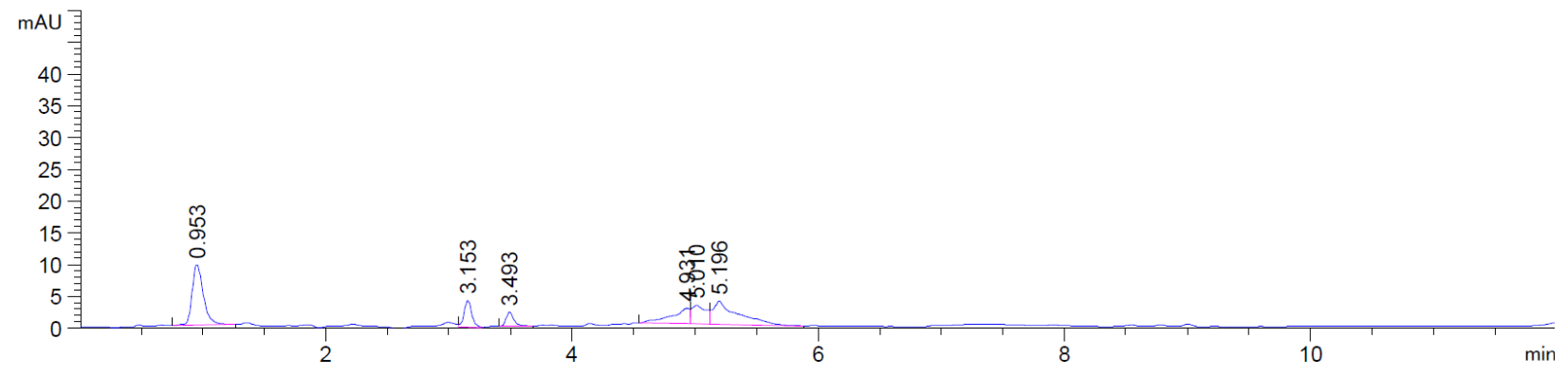


Reaction time $=90 \mathrm{~s}$

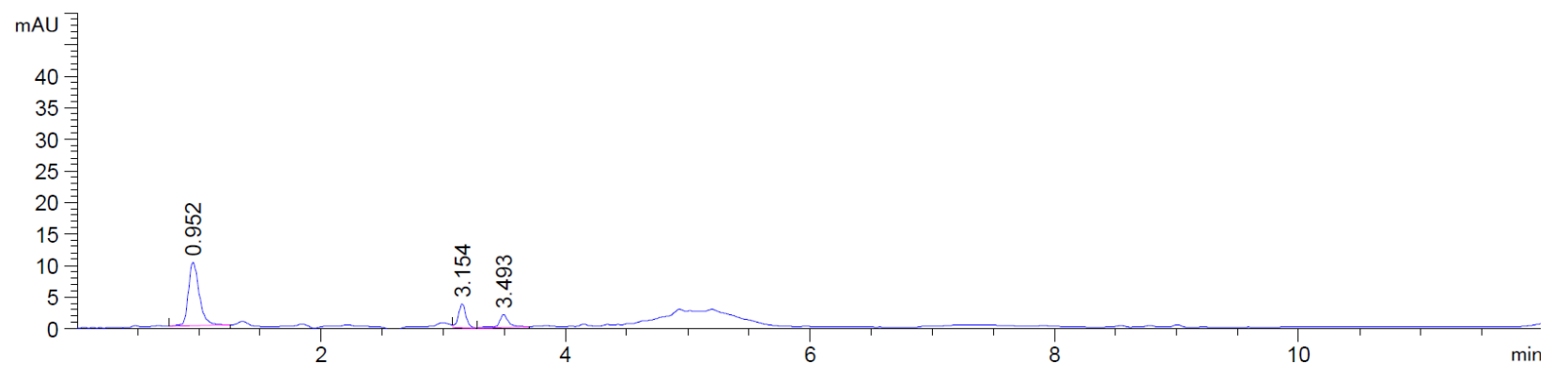


$(\mathrm{CyHQ})_{2}-\mathrm{O}, \mathrm{O}-\mathrm{DA}$ photolysis at $365 \mathrm{~nm}$

Reaction monitored at $280 \mathrm{~nm}$ for $\left.(\mathrm{CyHQ})_{2}-\mathrm{O}, \mathrm{O}-\mathrm{DA}\right)\left(t_{\mathrm{R}}=7.15 \mathrm{~min}\right), \mathrm{CyHQ}-\mathrm{O}-\mathrm{DA}\left(t_{\mathrm{R}}=4.99\right.$ and 5.18 $\mathrm{min})$, dopamine ( $\left.t_{\mathrm{R}}=0.95 \mathrm{~min}\right)$, and $\mathrm{CyHQ}-\mathrm{OH}\left(t_{\mathrm{R}}=3.1 \mathrm{~min}\right)$.

Reaction time $=0 \mathrm{~s}$

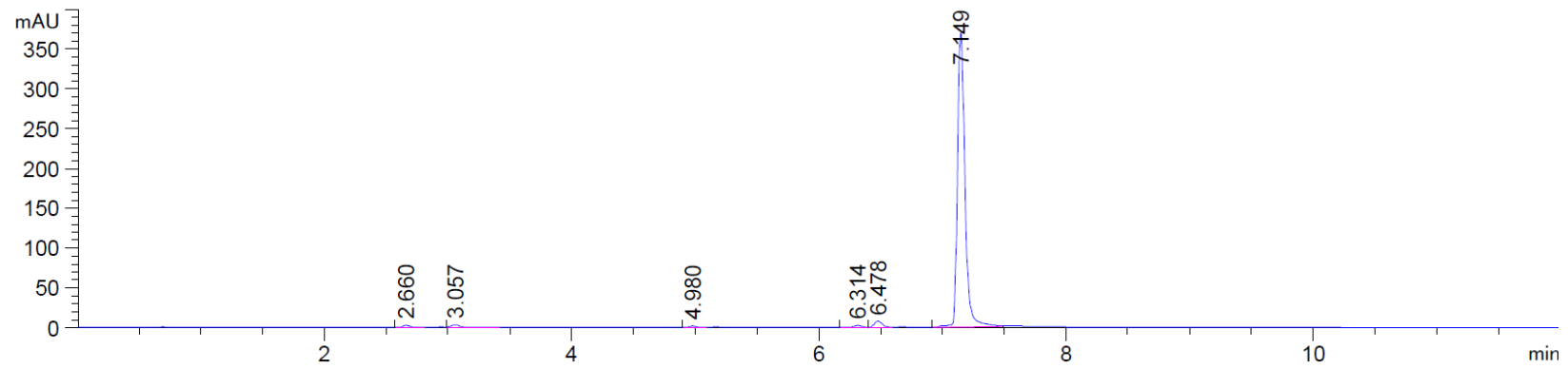

Reaction time $=5 \mathrm{~s}$

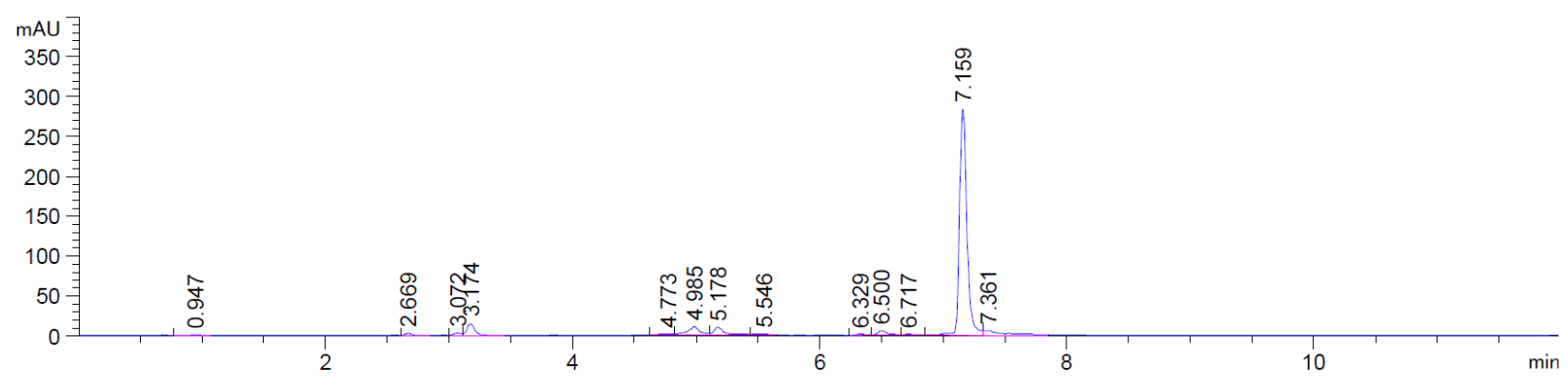

Reaction time $=30 \mathrm{~s}$

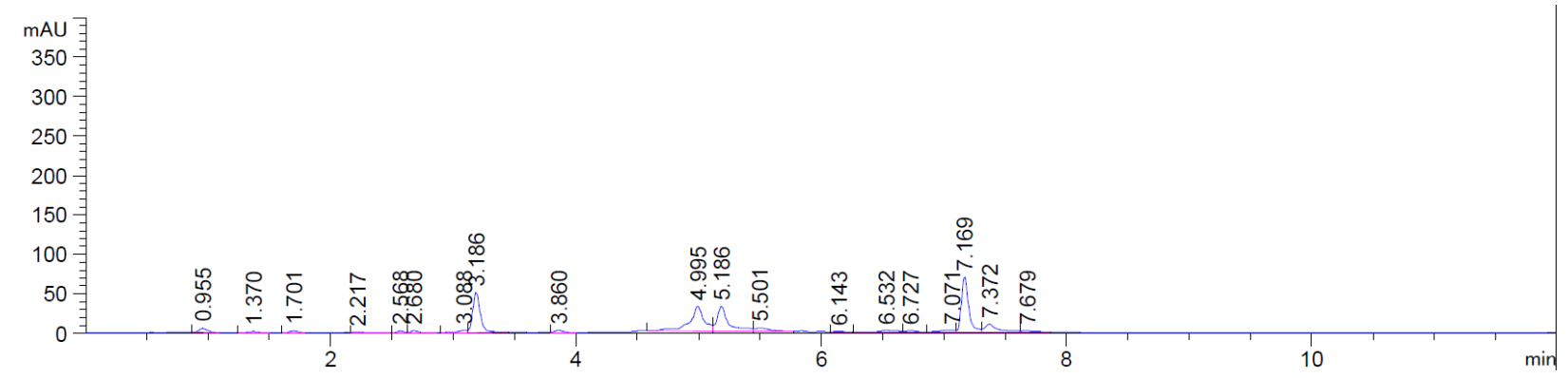

Reaction time $=90 \mathrm{~s}$

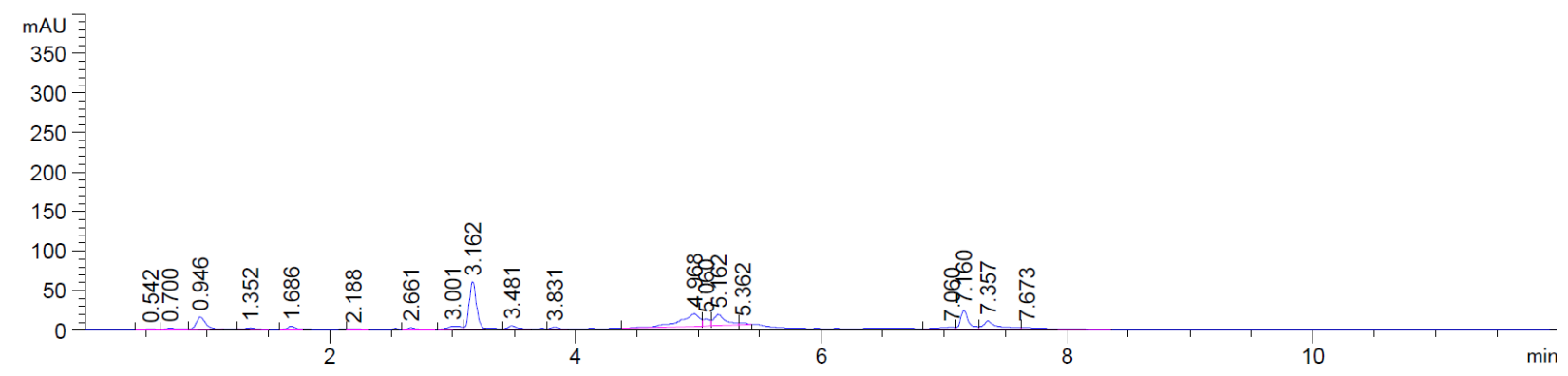


$(\text { CyHQ })_{2}-\mathrm{O}, \mathrm{O}-\mathrm{DA}$ photolysis at $405 \mathrm{~nm}$

Reaction monitored at $280 \mathrm{~nm}$ for $\left.(\mathrm{CyHQ})_{2}-\mathrm{O}, \mathrm{O}-\mathrm{DA}\right)\left(t_{\mathrm{R}}=7.15 \mathrm{~min}\right), \mathrm{CyHQ}-\mathrm{O}-\mathrm{DA}\left(t_{\mathrm{R}}=4.99\right.$ and 5.18 $\mathrm{min})$, dopamine ( $\left.t_{\mathrm{R}}=0.95 \mathrm{~min}\right)$, and $\mathrm{CyHQ}-\mathrm{OH}\left(t_{\mathrm{R}}=3.1 \mathrm{~min}\right)$.

\section{Reaction time $=0 \mathrm{~s}$}

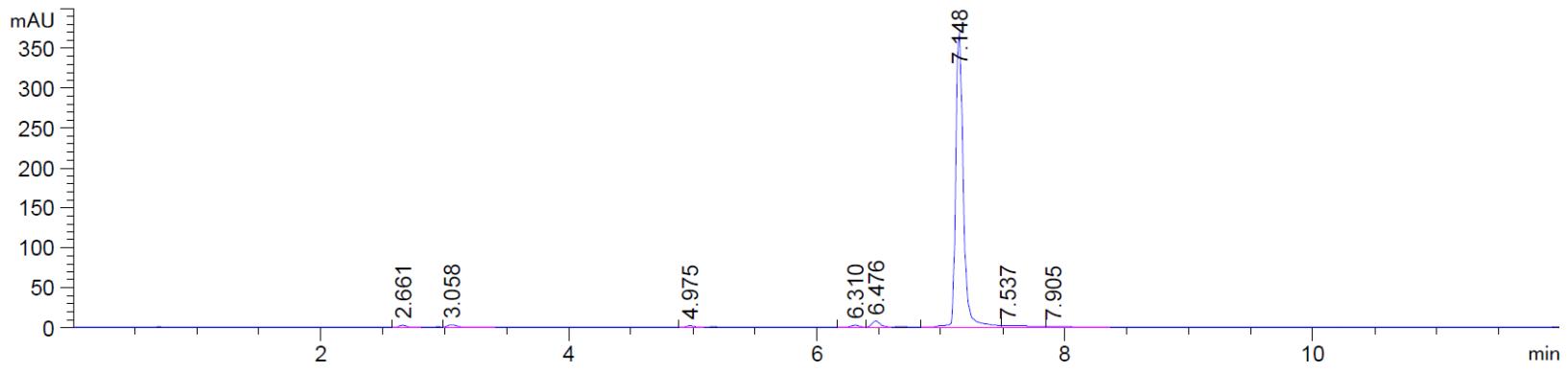

\section{Reaction time $=20 \mathrm{~s}$}

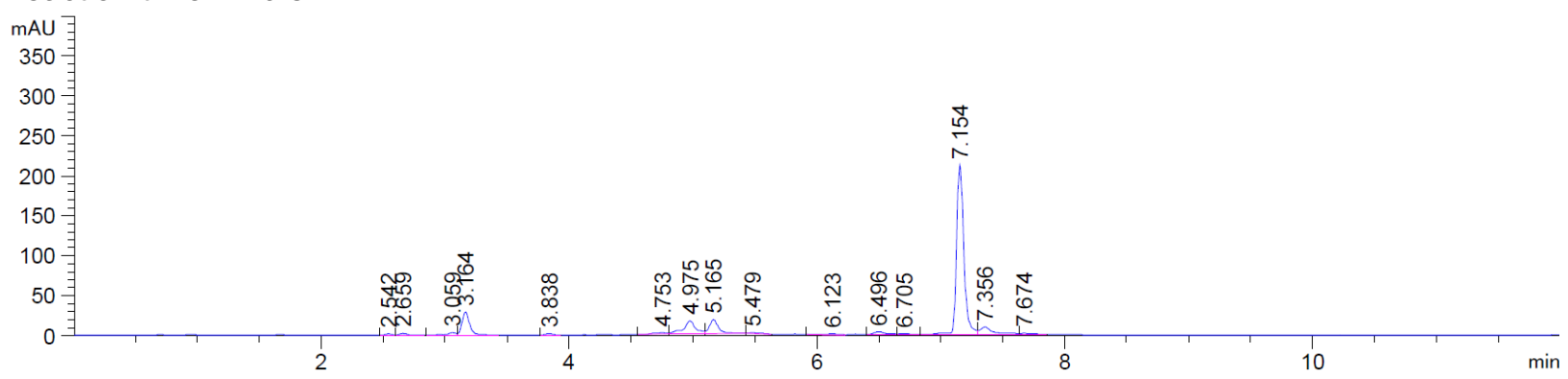

Reaction time $=40 \mathrm{~s}$

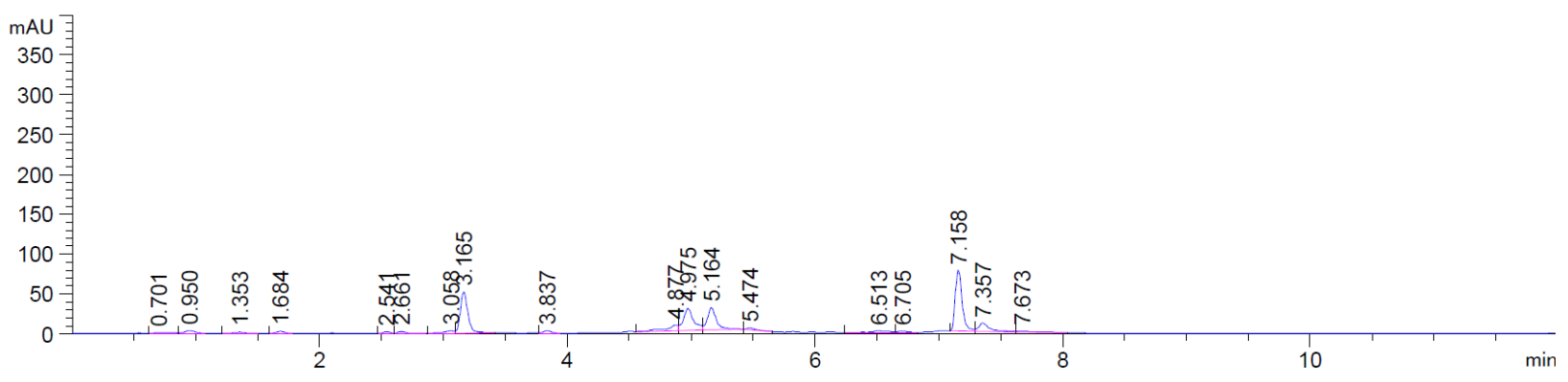

Reaction time $=90 \mathrm{~s}$

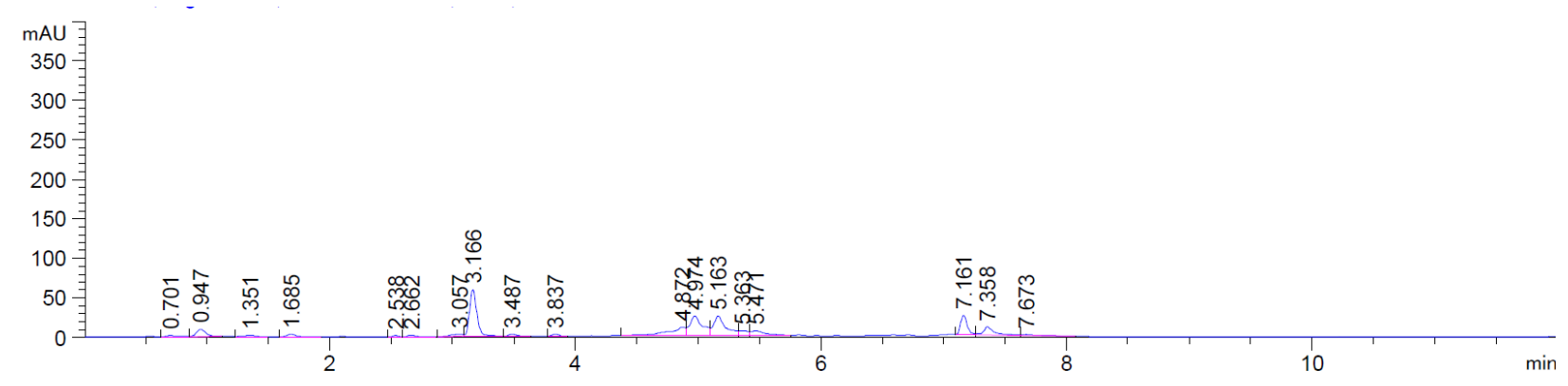


CyHQ-N-DA photolysis at $365 \mathrm{~nm}$

Reaction monitored at $280 \mathrm{~nm}$ for CyHQ- $N$-DA $\left(t_{\mathrm{R}}=6.45 \mathrm{~min}\right)$, dopamine $\left(t_{\mathrm{R}}=0.95 \mathrm{~min}\right)$, and CyHQ-OH $\left(t_{\mathrm{R}}=3.1 \mathrm{~min}\right)$.

Reaction time $=0 \mathrm{~s}$

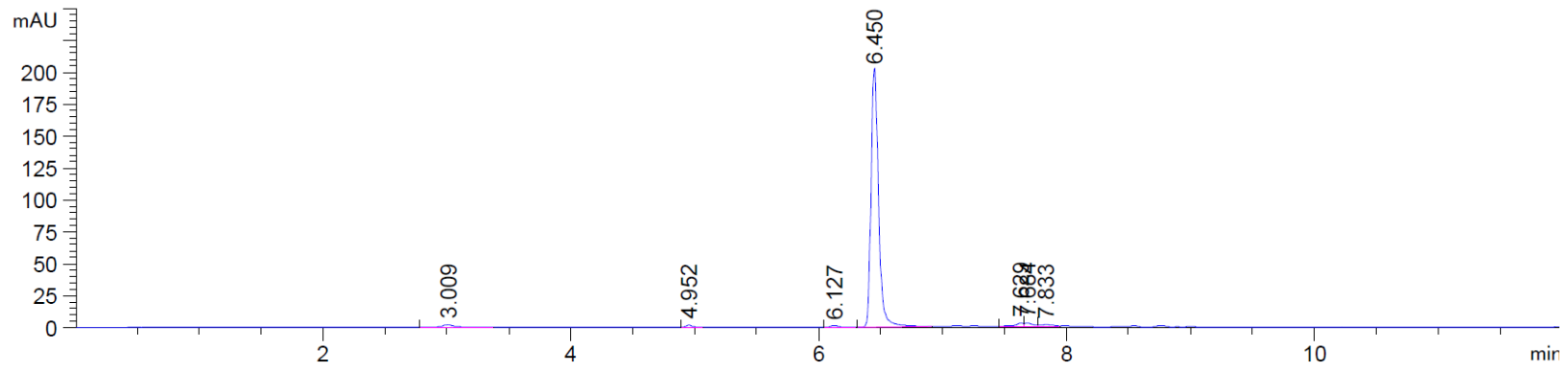

Reaction time $=5 \mathrm{~s}$

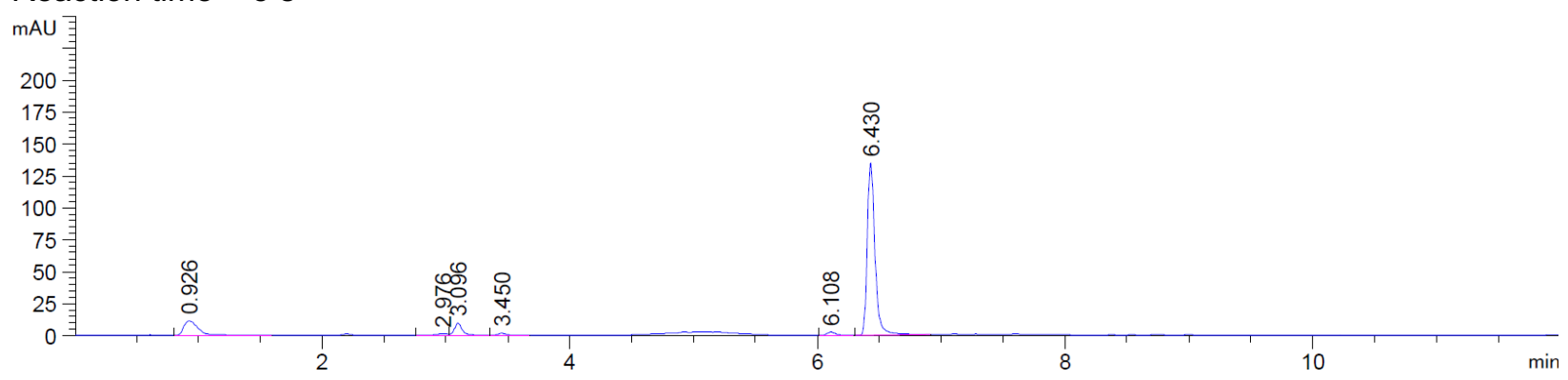

Reaction time $=30 \mathrm{~s}$

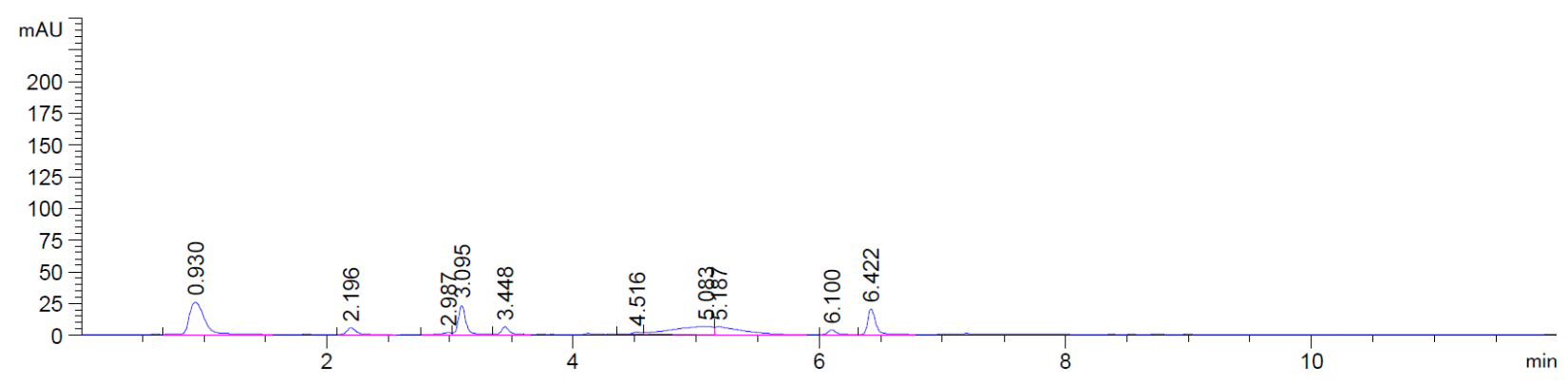

Reaction time $=60 \mathrm{~s}$

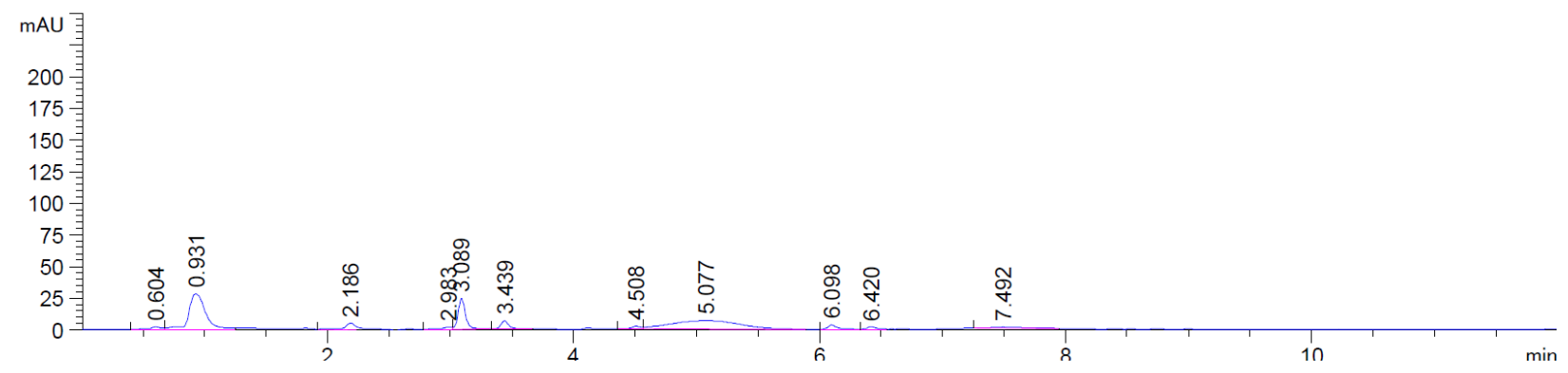


CyHQ-N-DA photolysis at $405 \mathrm{~nm}$

Reaction monitored at $280 \mathrm{~nm}$ for CyHQ- $N$-DA $\left(t_{\mathrm{R}}=6.45 \mathrm{~min}\right)$, dopamine $\left(t_{\mathrm{R}}=0.95 \mathrm{~min}\right)$, and CyHQ-OH $\left(t_{\mathrm{R}}=3.1 \mathrm{~min}\right)$.

Reaction time $=0 \mathrm{~s}$

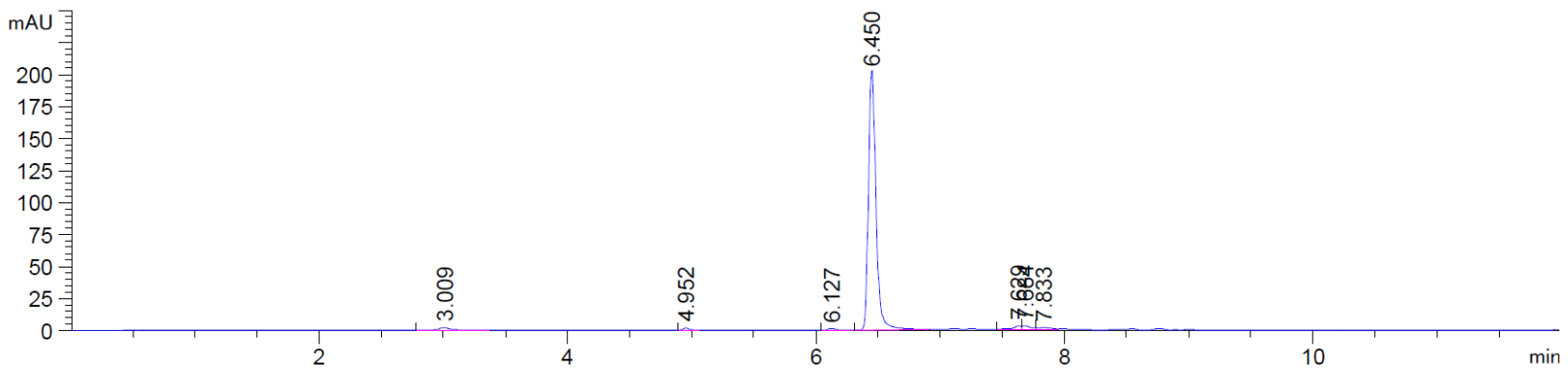

Reaction time $=30 \mathrm{~s}$

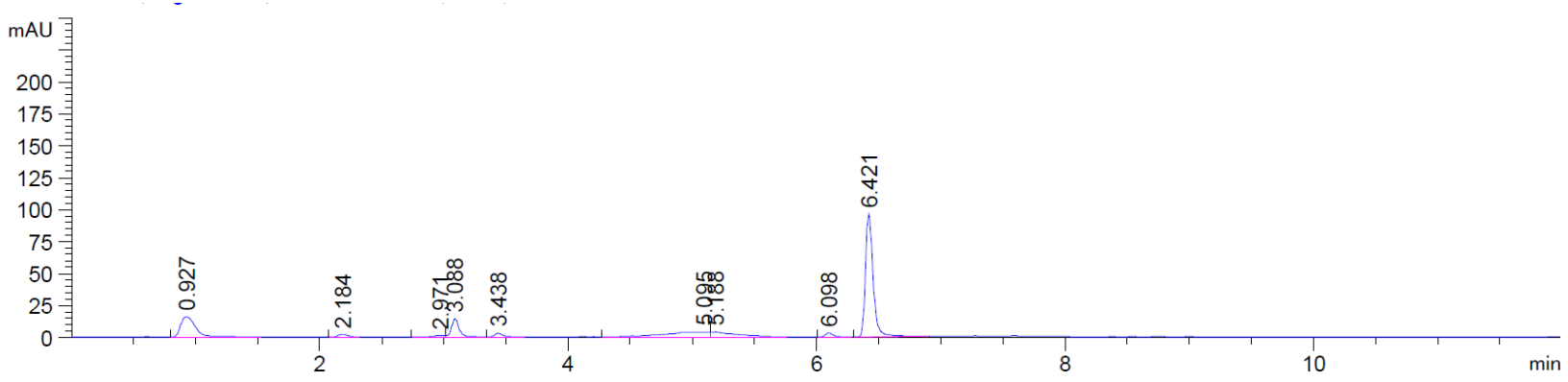

Reaction time $=90 \mathrm{~s}$

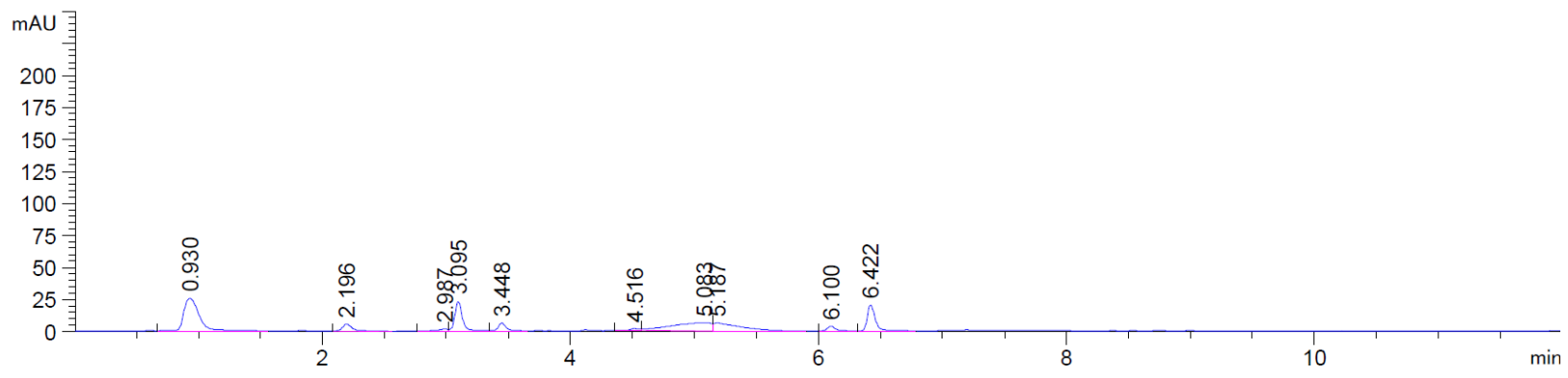

Reaction time $=180 \mathrm{~s}$

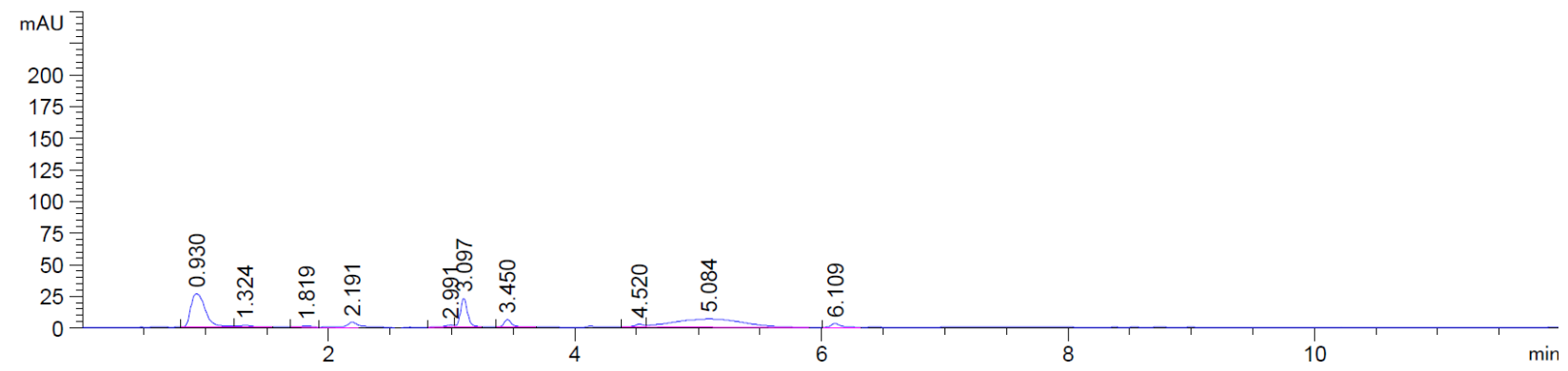


$(\mathrm{CyHQ})_{2}-\mathrm{N}, \mathrm{O}-\mathrm{DA}$ photolysis at $365 \mathrm{~nm}$

Reaction monitored at $280 \mathrm{~nm}$ for $\left((\mathrm{CyHQ})_{2}-\mathrm{N}, \mathrm{O}-\mathrm{DA}\right)\left(t_{\mathrm{R}}=7.64 \mathrm{~min}\right), \mathrm{CyHQ}-\mathrm{O}-\mathrm{DA}\left(t_{\mathrm{R}}=4.99\right.$ and 5.18 $\mathrm{min})$, CyHQ- $N$-DA ( $\left.t_{\mathrm{R}}=6.45 \mathrm{~min}\right)$, dopamine $\left(t_{\mathrm{R}}=0.95 \mathrm{~min}\right)$, and $\mathrm{CyHQ}-\mathrm{OH}\left(t_{\mathrm{R}}=3.1 \mathrm{~min}\right)$.

Reaction time $=0 \mathrm{~s}$

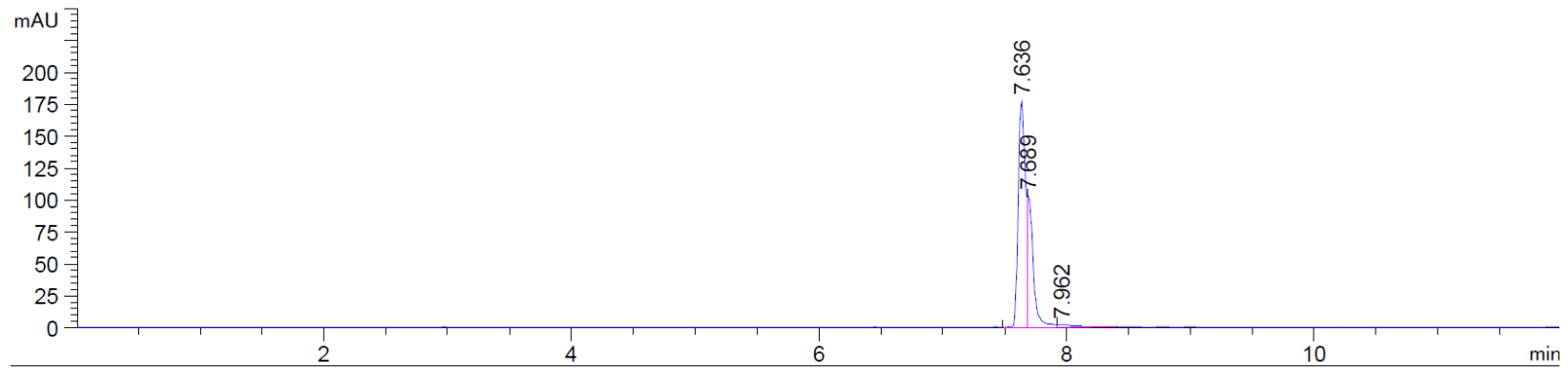

\section{Reaction time $=40 \mathrm{~s}$}

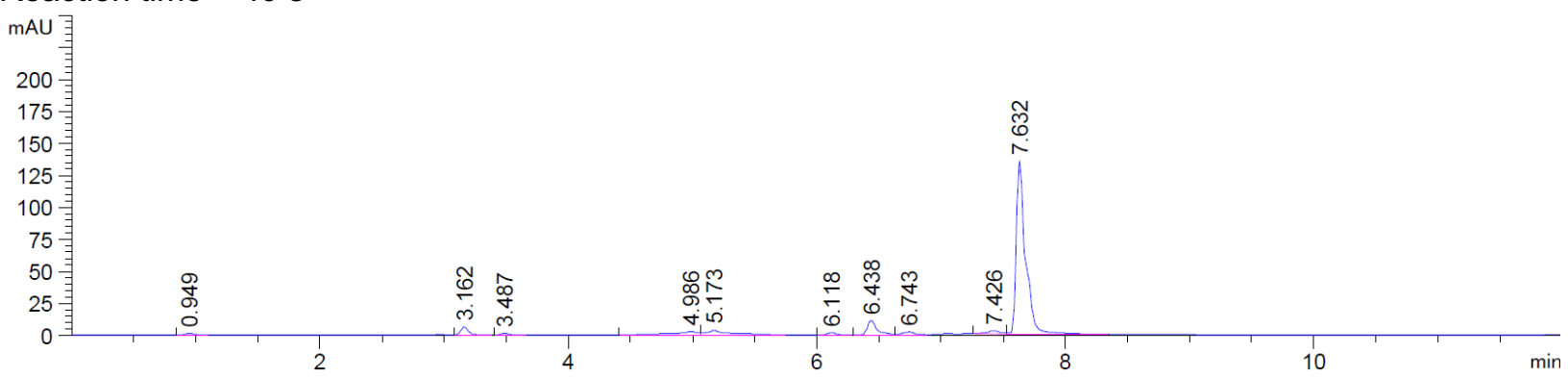

\section{Reaction time $=90 \mathrm{~s}$}
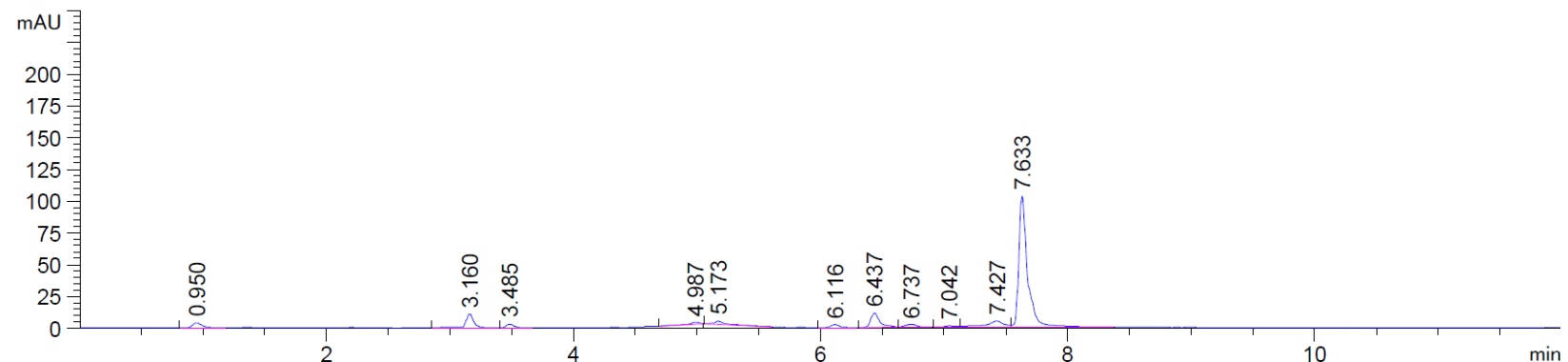

Reaction time $=180 \mathrm{~s}$
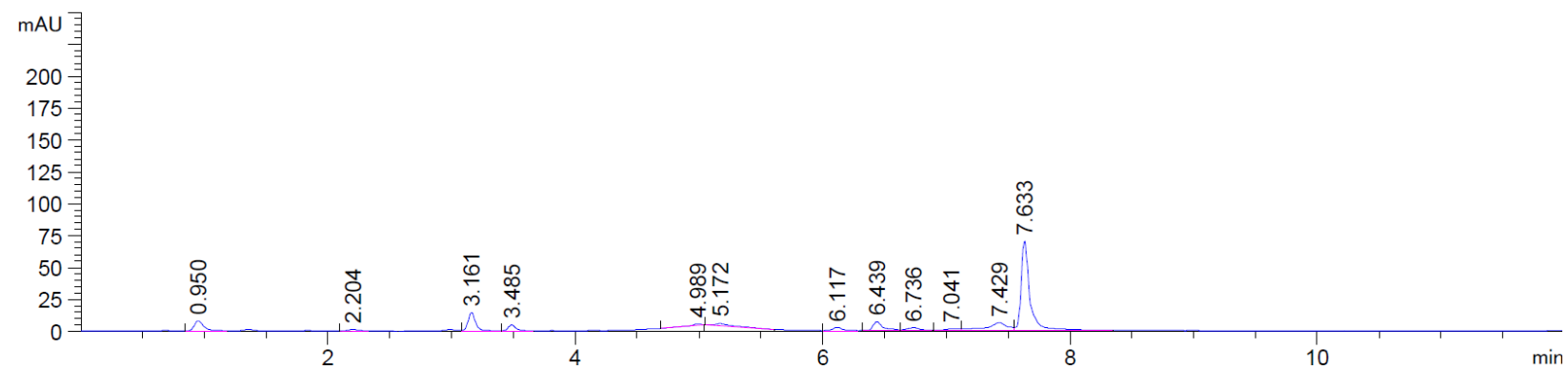
$(\mathrm{CyHQ})_{2}-\mathrm{N}, \mathrm{O}-\mathrm{DA}$ photolysis at $405 \mathrm{~nm}$

Reaction monitored at $280 \mathrm{~nm}$ for $\left((\mathrm{CyHQ})_{2}-\mathrm{N}, \mathrm{O}-\mathrm{DA}\right)\left(t_{\mathrm{R}}=7.64 \mathrm{~min}\right), \mathrm{CyHQ}-\mathrm{O}-\mathrm{DA}\left(t_{\mathrm{R}}=4.99\right.$ and 5.18 $\mathrm{min})$, CyHQ-N-DA ( $t_{\mathrm{R}}=6.45 \mathrm{~min}$, dopamine $\left(t_{\mathrm{R}}=0.95 \mathrm{~min}\right)$, and CyHQ-OH $\left(t_{\mathrm{R}}=3.1 \mathrm{~min}\right)$.

Reaction time $=0 \mathrm{~s}$

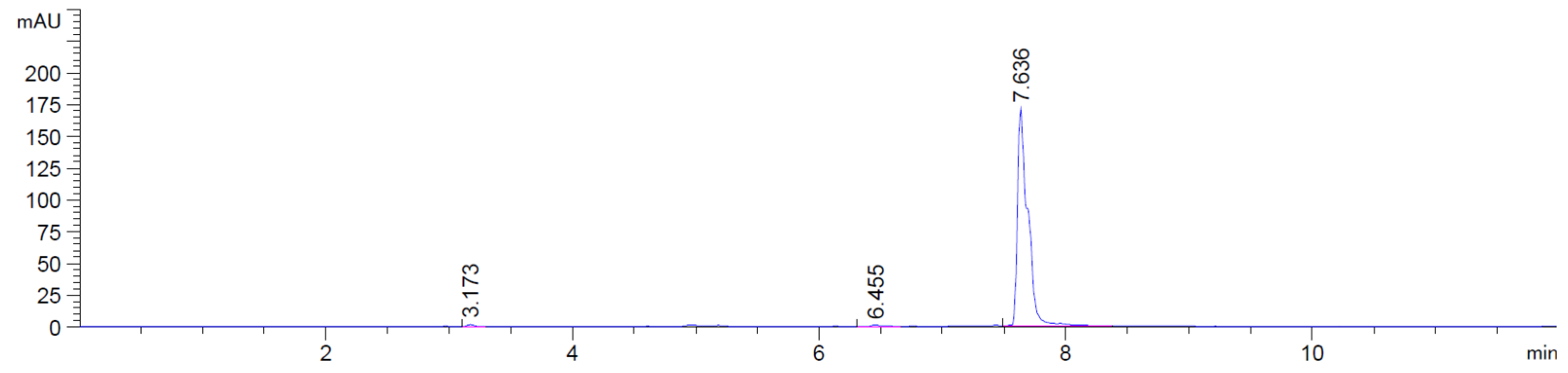

Reaction time $=300 \mathrm{~s}$

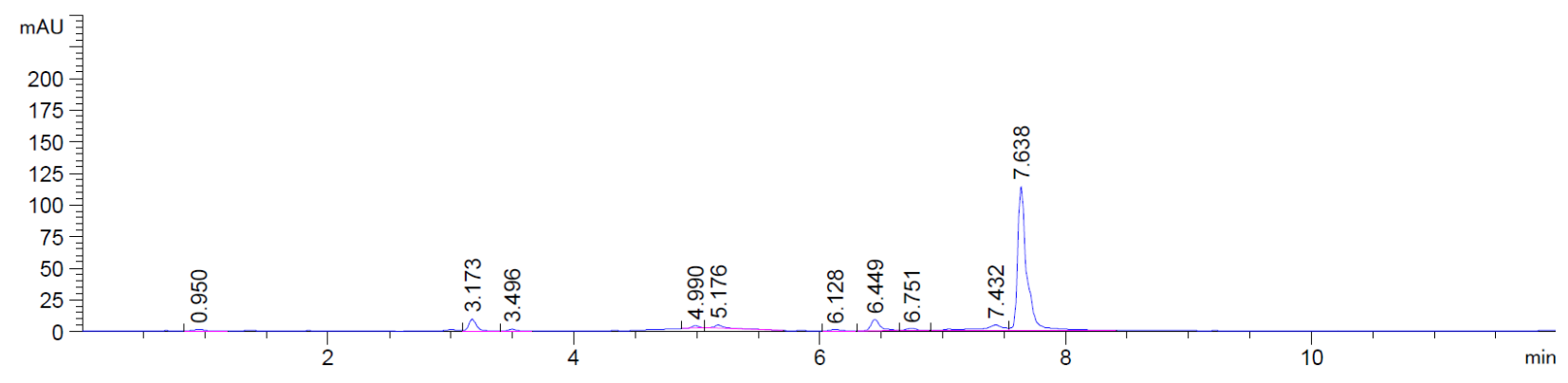


CyHQ-sulpiride photolysis at $365 \mathrm{~nm}$

Reaction monitored at $280 \mathrm{~nm}$ for CyHQ-sulpiride ( $t_{\mathrm{R}}=4.35$ and $4.63 \mathrm{~min}$ ), sulpiride $\left(t_{R}=2.25 \mathrm{~min}\right.$ ), and $\mathrm{CyHQ}-\mathrm{OH}\left(t_{\mathrm{R}}=3.1 \mathrm{~min}\right)$.

Reaction time $=0 \mathrm{~s}$

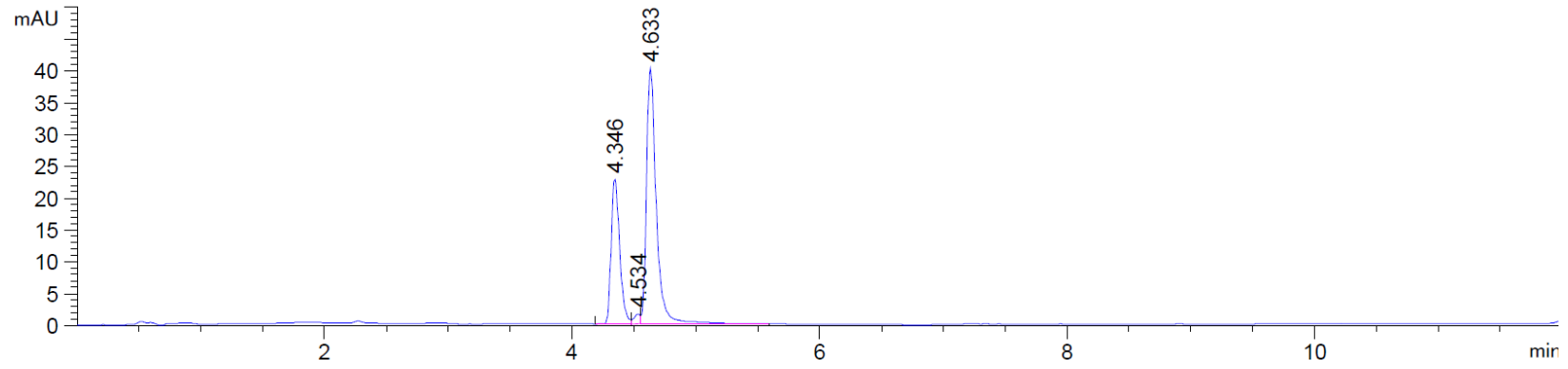

Reaction time $=5 \mathrm{~s}$
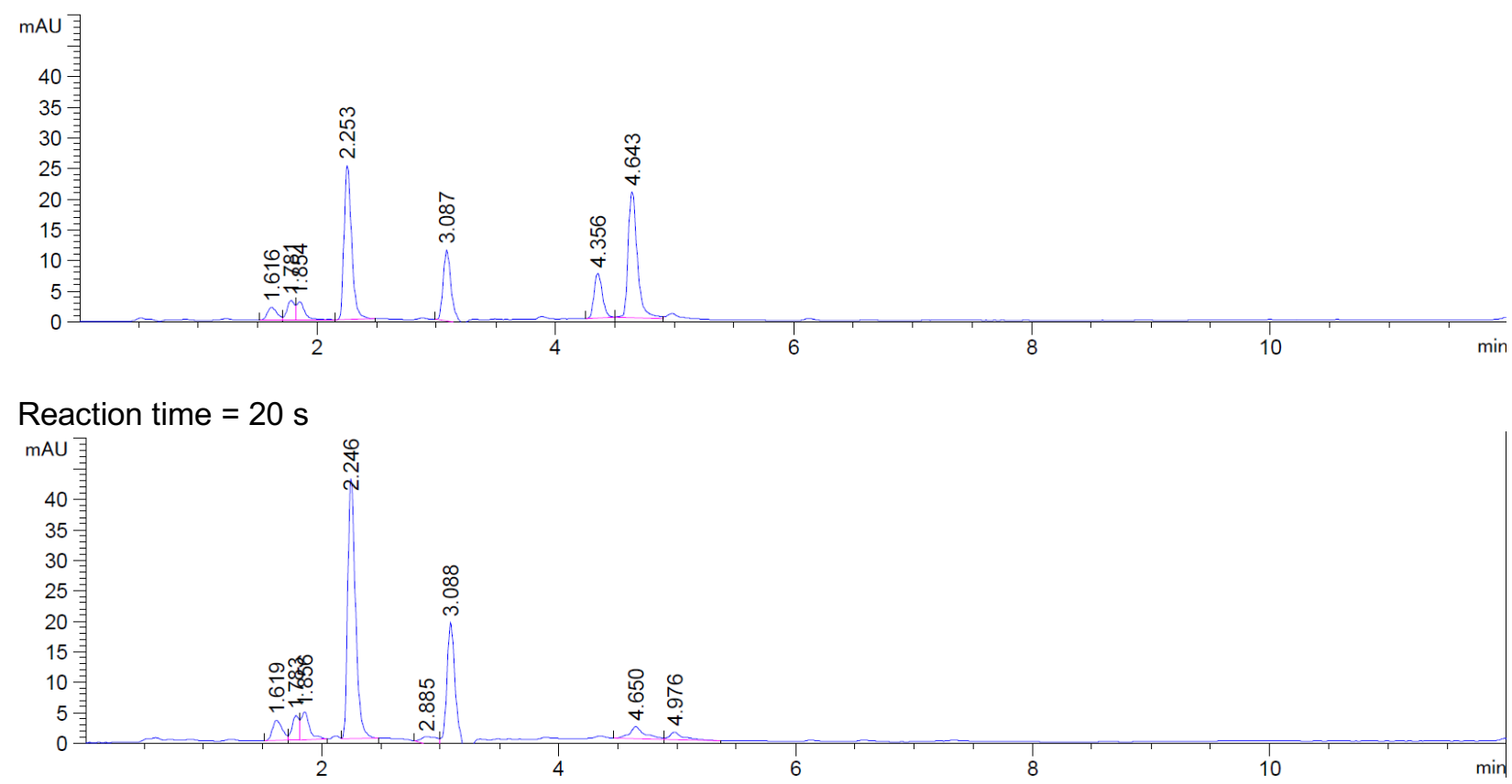

Reaction time $=60 \mathrm{~s}$

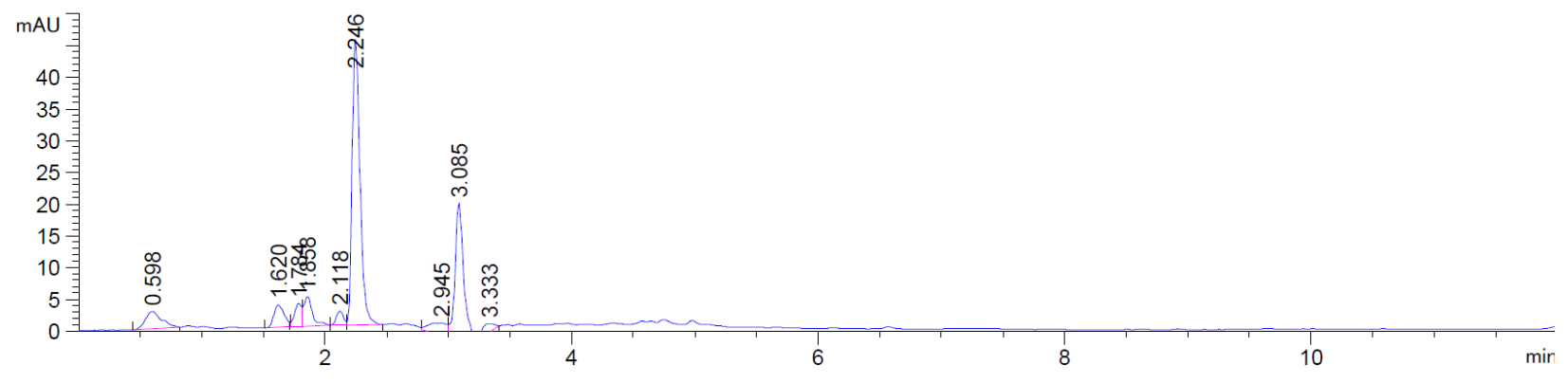


CyHQ-sulpiride photolysis at $405 \mathrm{~nm}$

Reaction monitored at $280 \mathrm{~nm}$ for CyHQ-sulpiride ( $t_{\mathrm{R}}=4.35$ and $4.63 \mathrm{~min}$ ), sulpiride $\left(t_{R}=2.25 \mathrm{~min}\right.$ ), and $\mathrm{CyHQ}-\mathrm{OH}\left(t_{\mathrm{R}}=3.1 \mathrm{~min}\right)$.

Reaction time $=0 \mathrm{~s}$

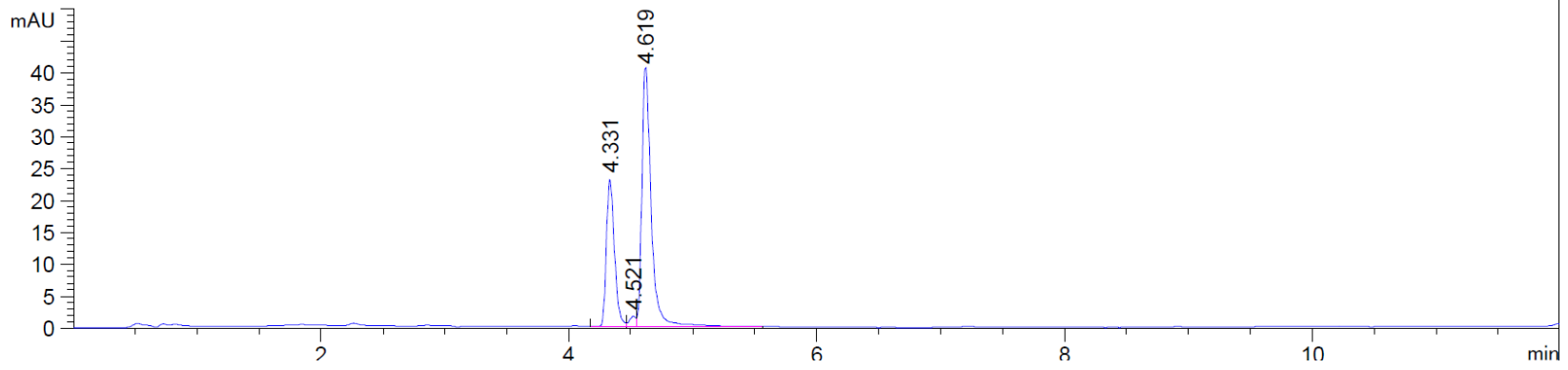

Reaction time $=5 \mathrm{~s}$

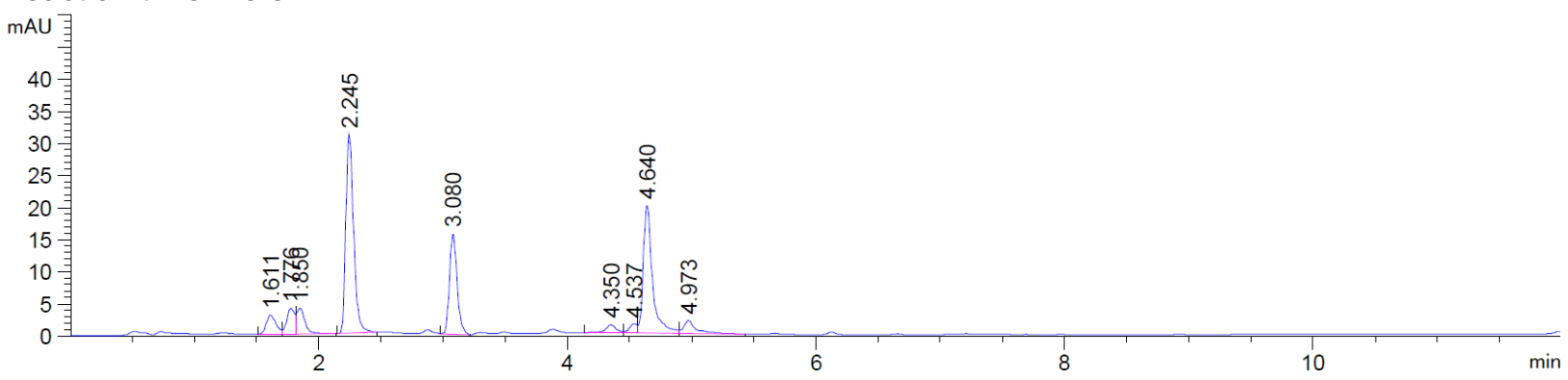

Reaction time $=30 \mathrm{~s}$

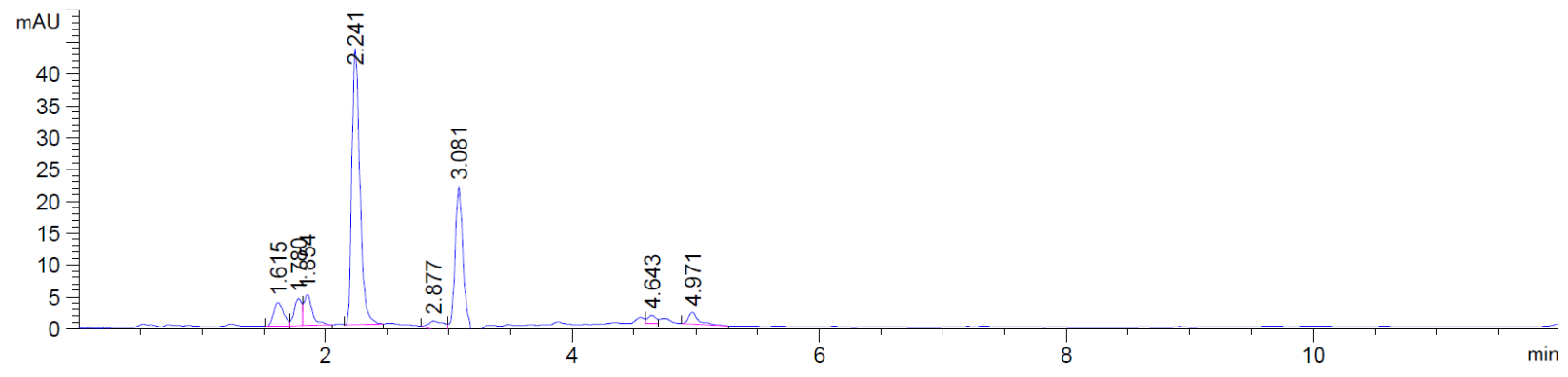

Reaction time $=90 \mathrm{~s}$

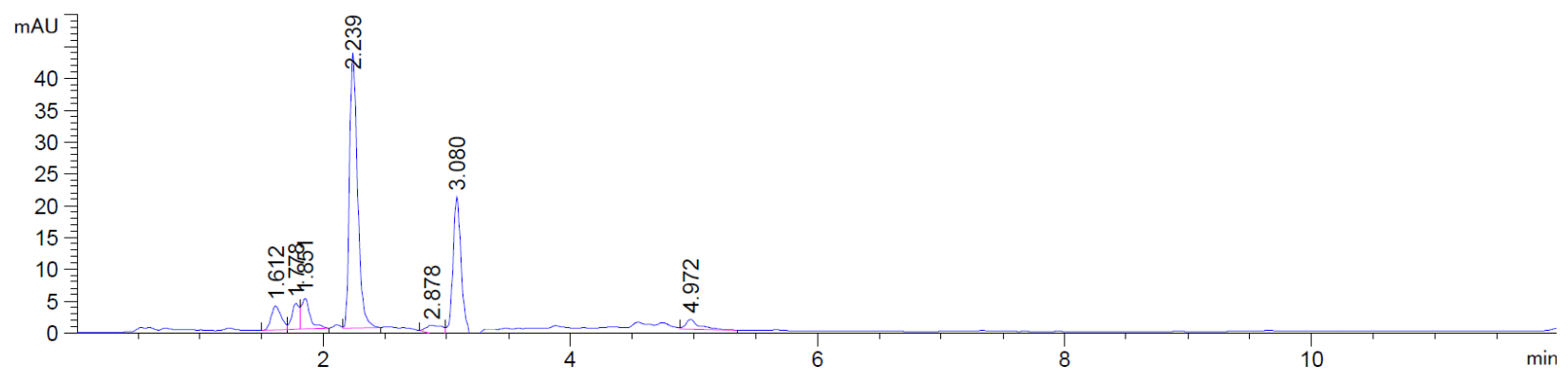




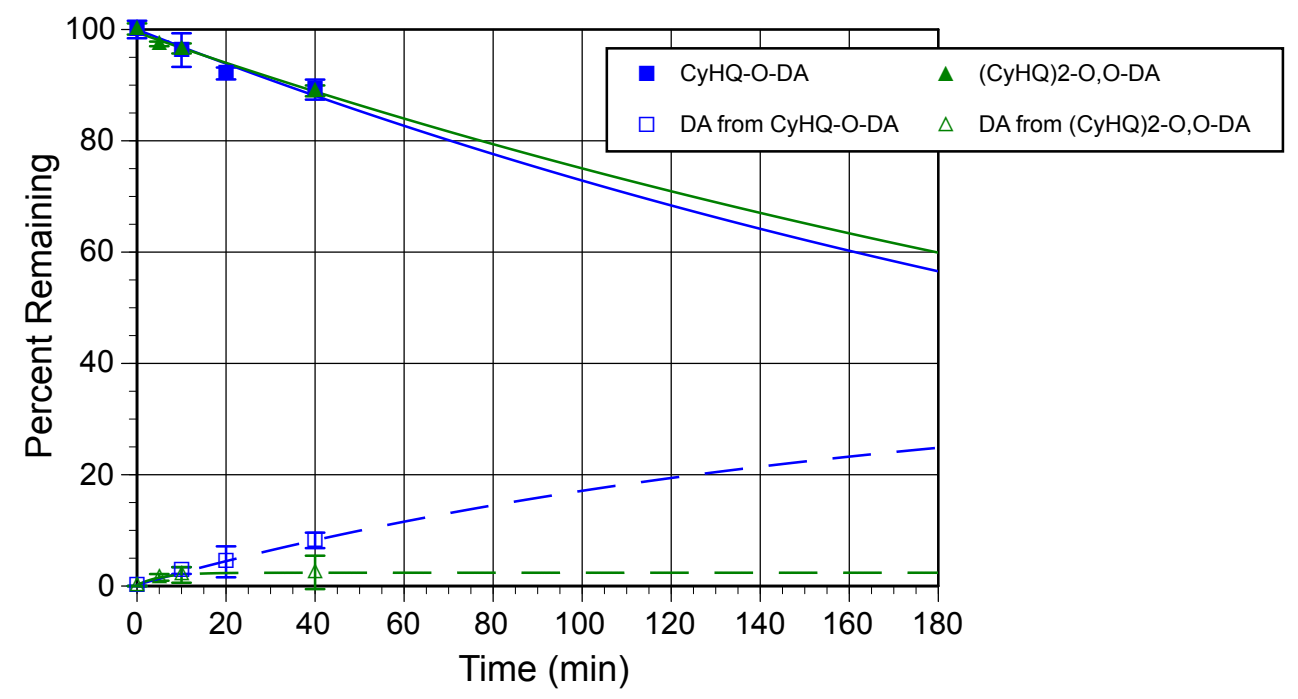

Figure S5. Time courses for the photoreaction of CyHQ-O-DA and (CyHQ)2-O,O-DA with 740-nm light (2PE) in KMOPS buffer.

Lines are the least-squares fit to an exponential decay for CyHQ-O-DA and an exponential rise to max for dopamine. Error bars represent the standard deviation of the average of three measurements.

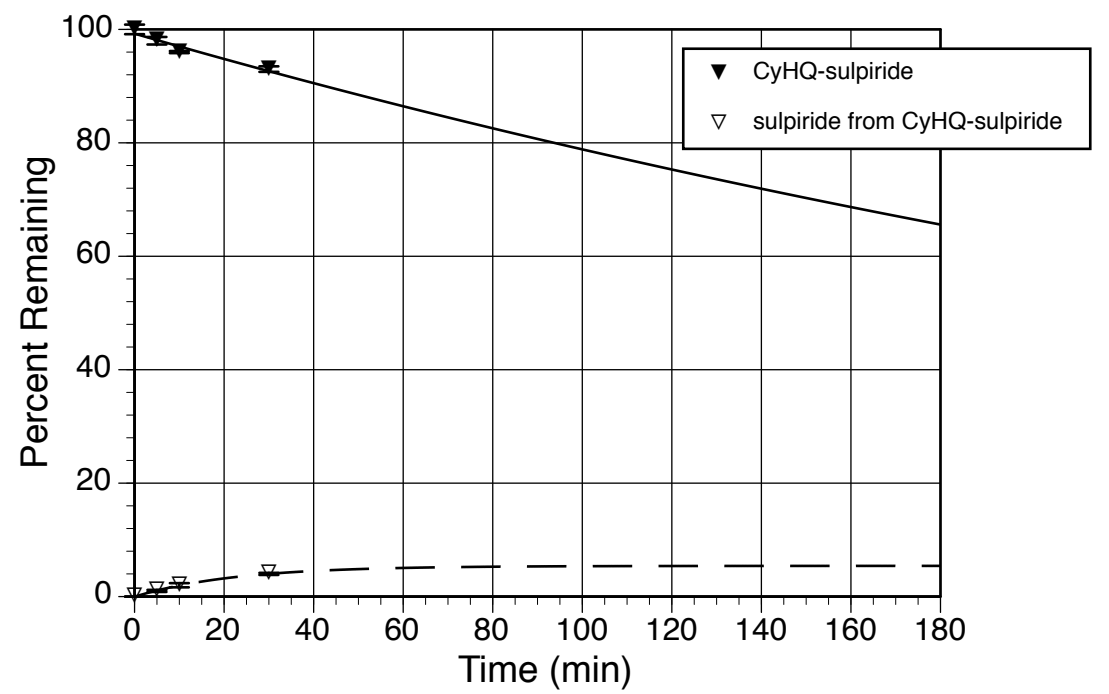

Figure S6. Time course for the photoreaction of CyHQ-sulpiride with 740-nm light (2PE) in KMOPS buffer.

Lines are the least-squares fit to an exponential decay for CyHQ-sulpiride and an exponential rise to max for sulpiride. Error bars represent the standard deviation of the average of three measurements. 
Table S2. Data for the calculation of 2-photon uncaging action cross-sections $\left(\delta_{u}\right)$

The 2-photon uncaging action cross section $\left(\delta_{u}\right)$ in Goeppert Mayer $\left(\mathrm{GM}, 10^{-50} \mathrm{~cm}^{4} \cdot \mathrm{s} /\right.$ photon) was determined using the following equation: ${ }^{3}$

$\delta_{u}=\frac{N_{p} \phi Q_{f 2} \delta_{a F} C_{F}}{<F(t)>C_{S}}$

$N_{p}=$ number of molecules photolyzed per second determined by HPLC analysis

$\phi=$ estimated collection efficiency of the fluorescence detector

$$
\begin{aligned}
& \phi=\frac{A y}{4 \pi R^{2} n^{2}} \\
& A=\text { area of detector }\left(0.33 \mathrm{~cm}^{2}\right) \\
& y=\text { fraction of integrated emission spectrum transmitted by interference filter (0.465) } \\
& R=\text { distance from the center of the cuvette to the detector } \\
& n=\text { refractive index of water (1.33) }
\end{aligned}
$$

$Q_{\mathfrak{f} 2}=$ fluorescence quantum yield of the external standard fluorescein $(0.9)^{6,7}$

$\delta_{\mathrm{aF}}=2$-photon absorption cross section of fluorescein $(30 \mathrm{GM} \text { at } 740 \mathrm{~nm})^{8}$

\begin{tabular}{|c|c|c|c|c|c|c|}
\hline Compound & $\begin{array}{c}C_{F} \\
(\mu \mathrm{M})\end{array}$ & $\begin{array}{c}C_{s} \\
(\mu \mathrm{M})\end{array}$ & $\begin{array}{c}R \\
(\mathrm{~cm})\end{array}$ & $N_{p}$ & $\phi$ & $\begin{array}{c}\langle\mathrm{F}(\mathrm{t})\rangle \\
\text { (photons/s) }\end{array}$ \\
\hline CyHQ-O-DA & 12 & 104 & 2.25 & $3.81 \times 10^{11}$ & $1.36 \times 10^{-3}$ & $6.65 \times 10^{9}$ \\
\hline$(\mathrm{CyHQ})_{2}-\mathrm{O}, \mathrm{O}-\mathrm{DA}$ & 10 & 100 & 2.86 & $6.90 \times 10^{10}$ & $8.40 \times 10^{-4}$ & $1.28 \times 10^{9}$ \\
\hline CyHQ-sulpiride & 10 & 100 & 2.86 & $4.39 \times 10^{10}$ & $8.40 \times 10^{-4}$ & $3.88 \times 10^{8}$ \\
\hline
\end{tabular}

$\mathrm{C}_{\mathrm{F}}=$ concentration of fluorescein

$\langle\mathrm{F}(\mathrm{t})\rangle=$ time-averaged fluorescence photon flux of the fluorescein standard measured by a radiometer positioned at a right angle to the excitation laser beam (photons/s)

$\mathrm{C}_{\mathrm{s}}=$ concentration of sample being photolyzed 
A

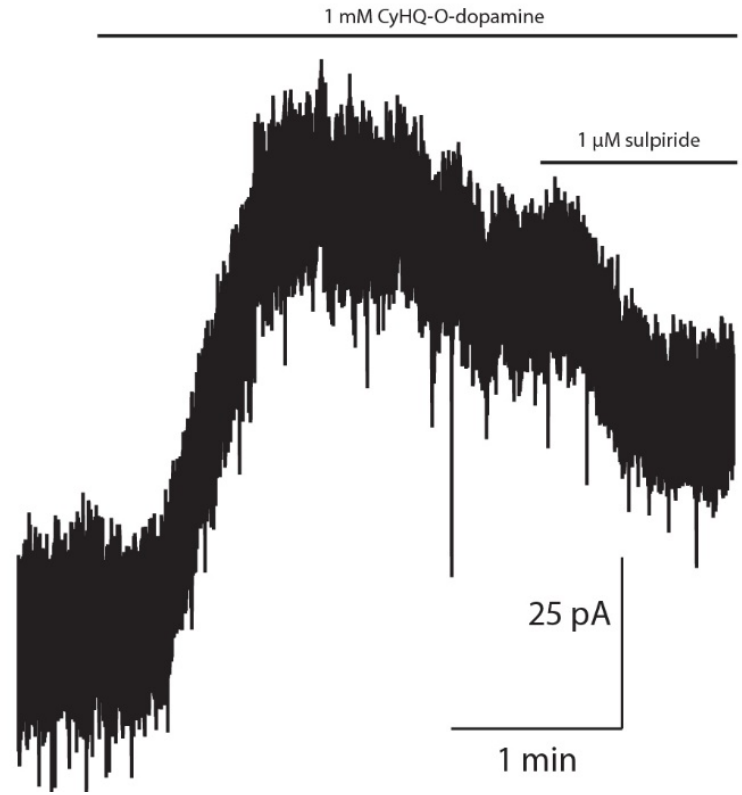

B

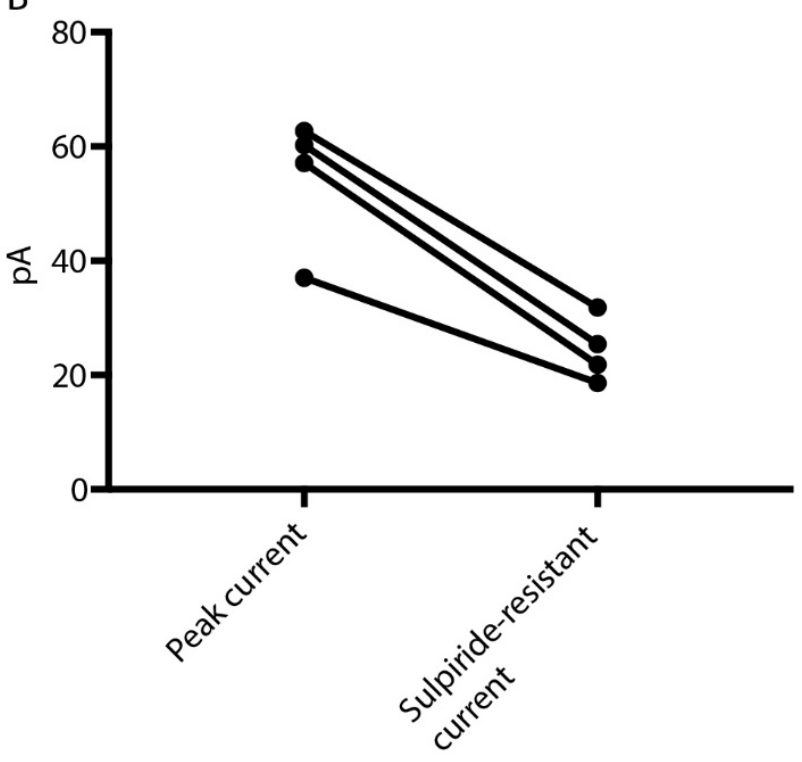

Figure S7. The current produced by CyHQ-O-DA $(1 \mathrm{mM})$ is not fully blocked by sulpiride.

(A) Example trace produced from superperfusion of $1 \mathrm{mM}$ CyHQ-O-dopamine in the dark. The current elicited reaches a peak in about a minute then the D2 response begins to desensitize. Antagonism with sulpiride $(1 \mu \mathrm{M})$ only partially reverses the current, suggesting it is not due to a low concentration of free dopamine. (B) Summary data for the experiment depicted in (A). The peak current is matched with the remaining shift in baseline current after application of sulpiride.

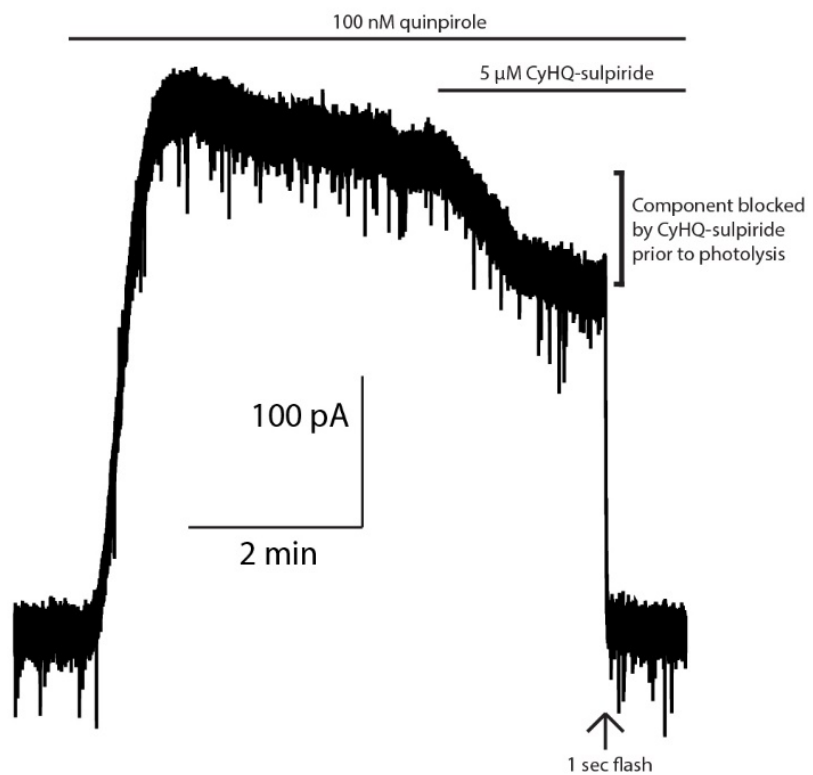

Figure S8. CyHQ-sulpiride alone has a small level of antagonism prior to photolysis.

Quinpirole $(100 \mathrm{nM})$ was recirculated at a low concentration, picked to emphasize any partial receptor block. CyHQ-sulpride $(5 \mu \mathrm{M})$ was then added and a small amount of antagonism can be observed by an inward deflection of the outward current prior to photolysis. 


\section{${ }^{1} \mathrm{H}$ and ${ }^{13} \mathrm{C}$ NMR Spectra}

tert-Butyl (3-((8-cyano-7-(methoxymethoxy)quinolin-2-yl)methoxy)-4-hydroxyphenethyl)carbamate and tert-Butyl (4-((8-cyano-7-(methoxymethoxy)quinolin-2-yl)methoxy)-3-hydroxyphenethyl)carbamate (MOMCyHQ-O-DA-Boc)

${ }^{1} \mathrm{H}$ NMR $(600 \mathrm{MHz}$, chloroform-d)

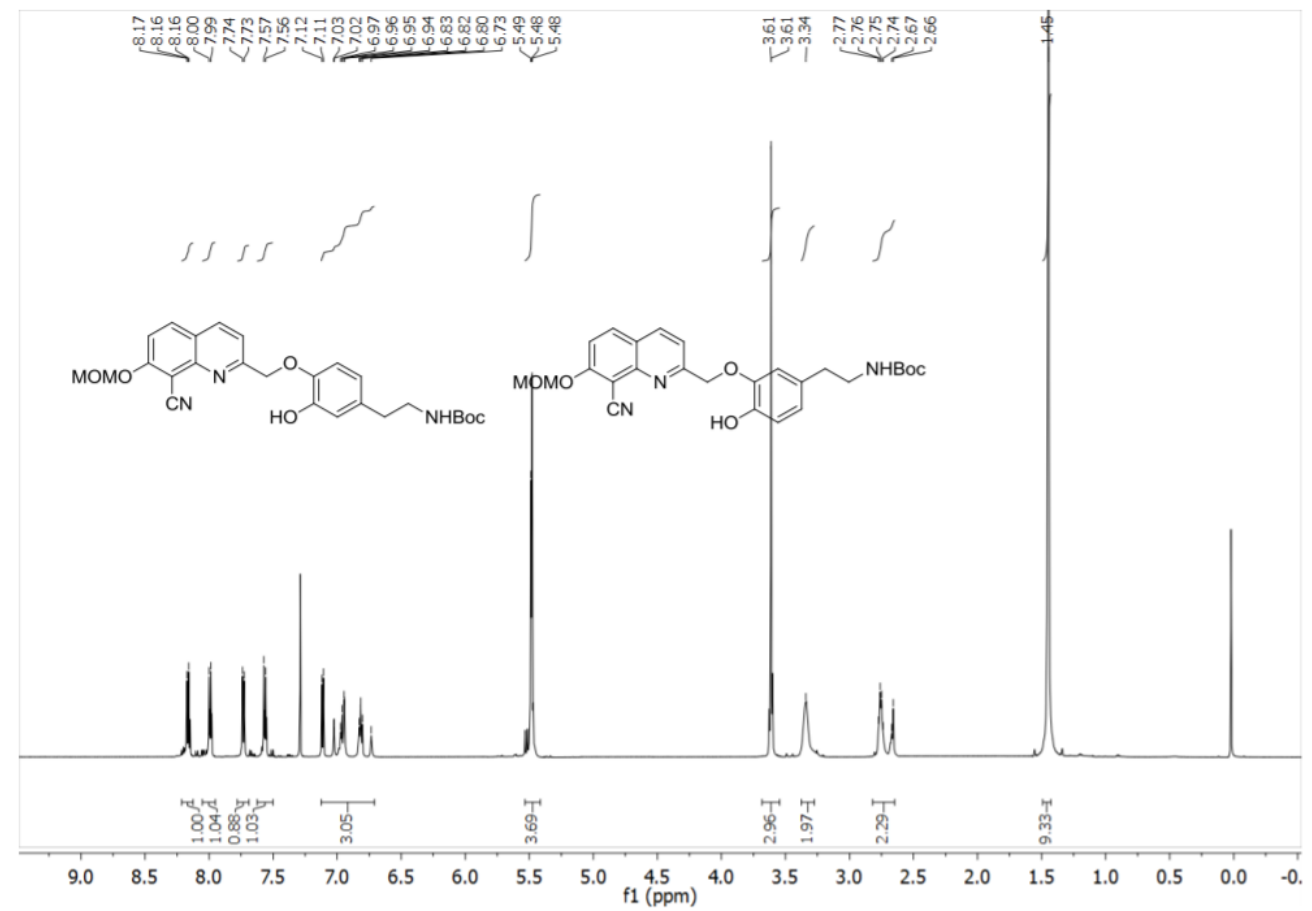

${ }^{13} \mathrm{C}$ NMR $\left(151 \mathrm{MHz}\right.$, methanol- $\left.d_{4}\right)$

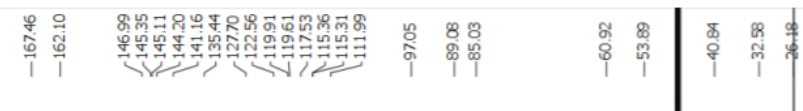

$\begin{array}{lllllllllllllllllllllll}210 & 200 & 190 & 180 & 170 & 160 & 150 & 140 & 130 & 120 & 110_{\mathrm{f} 1(\mathrm{ppm})}^{100} & 90 & 80 & 70 & 60 & 50 & 40 & 30 & 20 & 10 & 0 & -10\end{array}$ 
2-(3-((8-cyano-7-hydroxyquinolin-2-yl)methoxy)-4-hydroxyphenyl)ethan-1-aminium 2, 2, 2-trifluoroacetate and 2-(4-((8-cyano-7-hydroxyquinolin-2-yl)methoxy)-3-hydroxyphenyl)ethan-1-aminium 2, 2, 2trifluoroacetate (CyHQ-O-DA).

${ }^{1} \mathrm{H}$ NMR $\left(500 \mathrm{MHz}\right.$, methanol- $\left.d_{4}\right)$

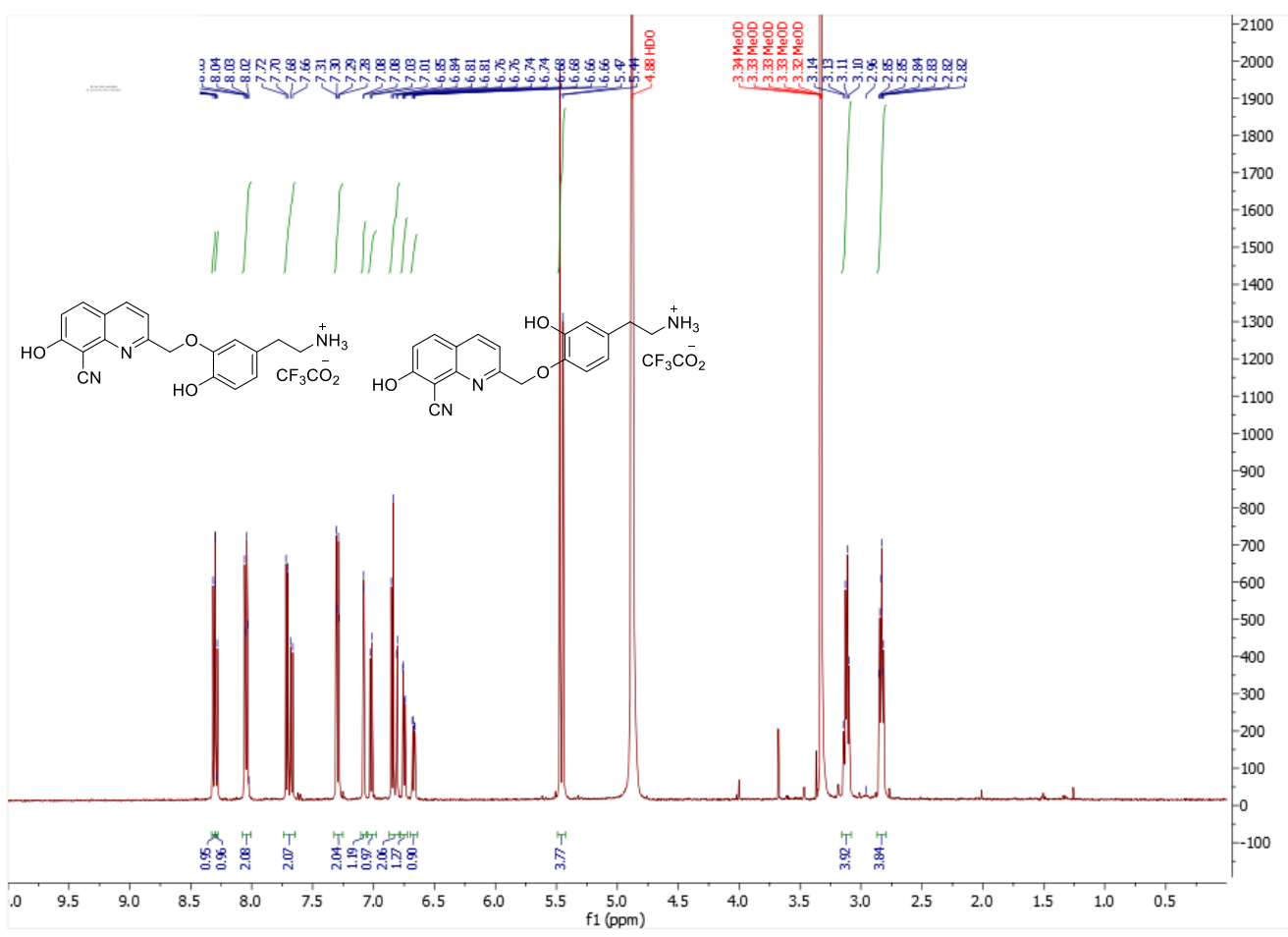

${ }^{13} \mathrm{C}$ NMR (126 MHz, methanol- $\left.d_{4}\right)$

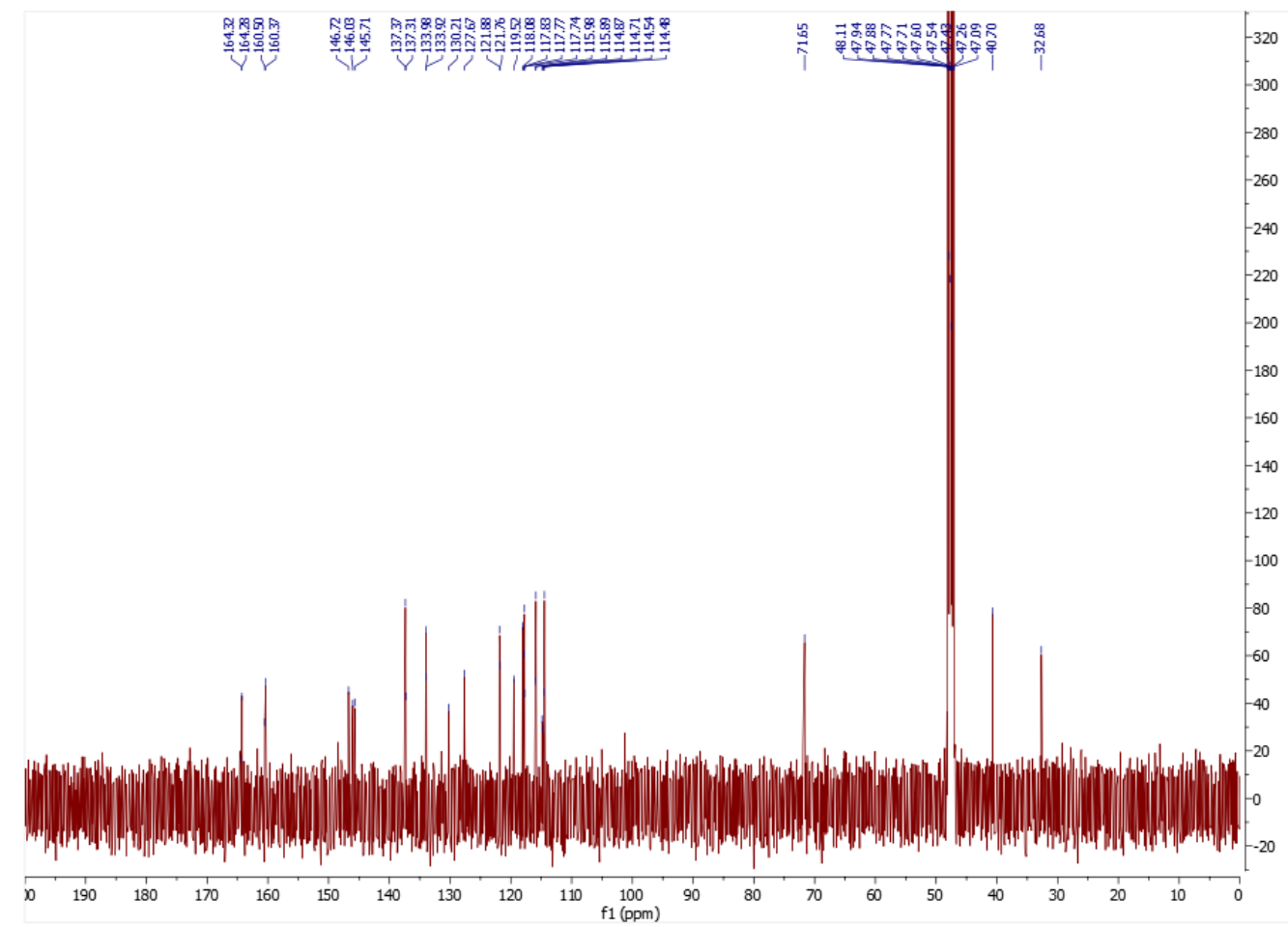


2-(3,4-bis((8-cyano-7-hydroxyquinolin-2-yl)methoxy)phenyl)ethan-1-aminium 2,2,2-trifluoroacetate $\left((C y H Q)_{2}-0,0-D A\right)$

${ }^{1} \mathrm{H}$ NMR $\left(500 \mathrm{MHz}\right.$, methanol- $\left.d_{4}\right)$

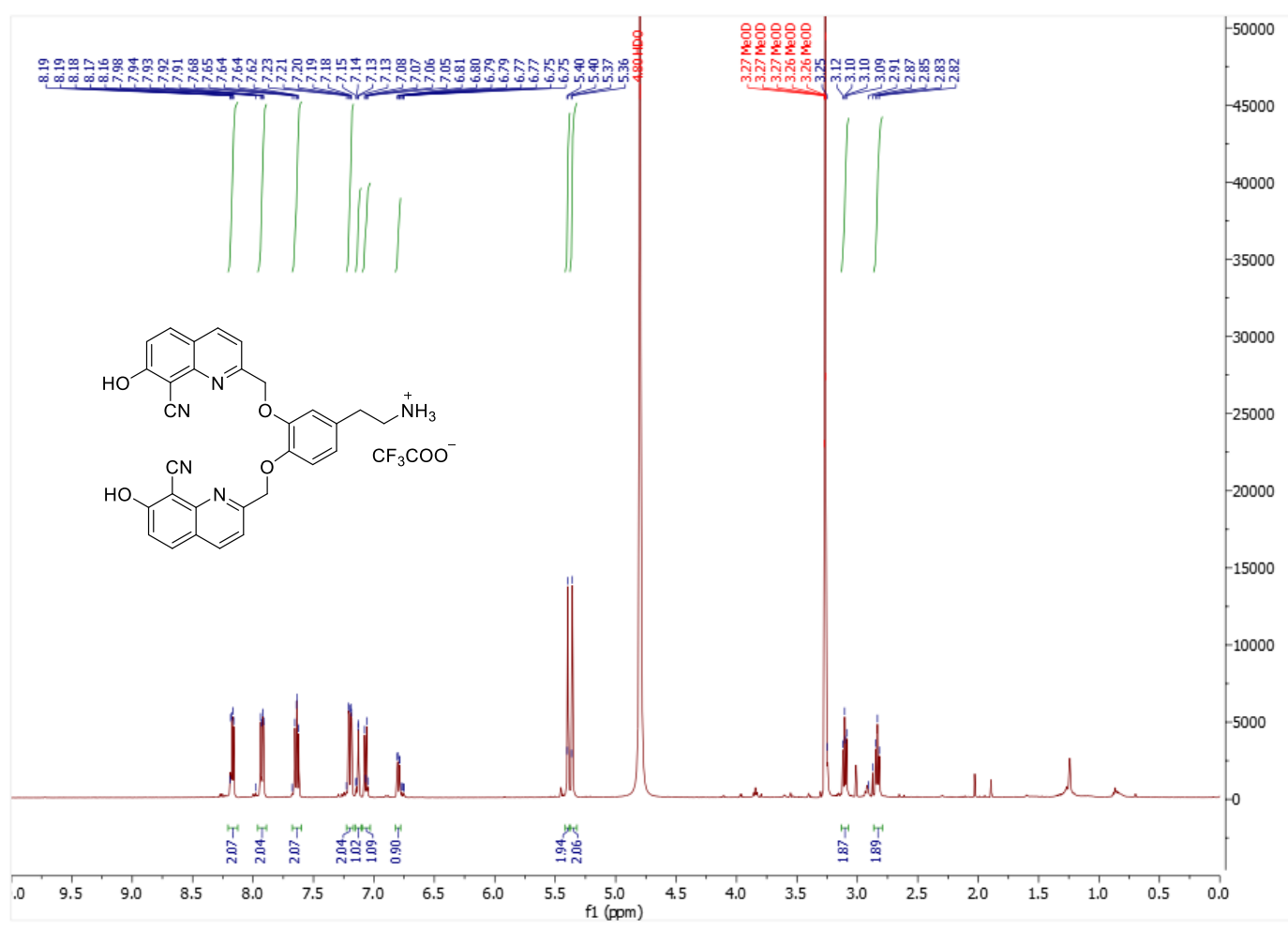

${ }^{13} \mathrm{C}$ NMR (126 MHz, methanol- $\left.d_{4}\right)$

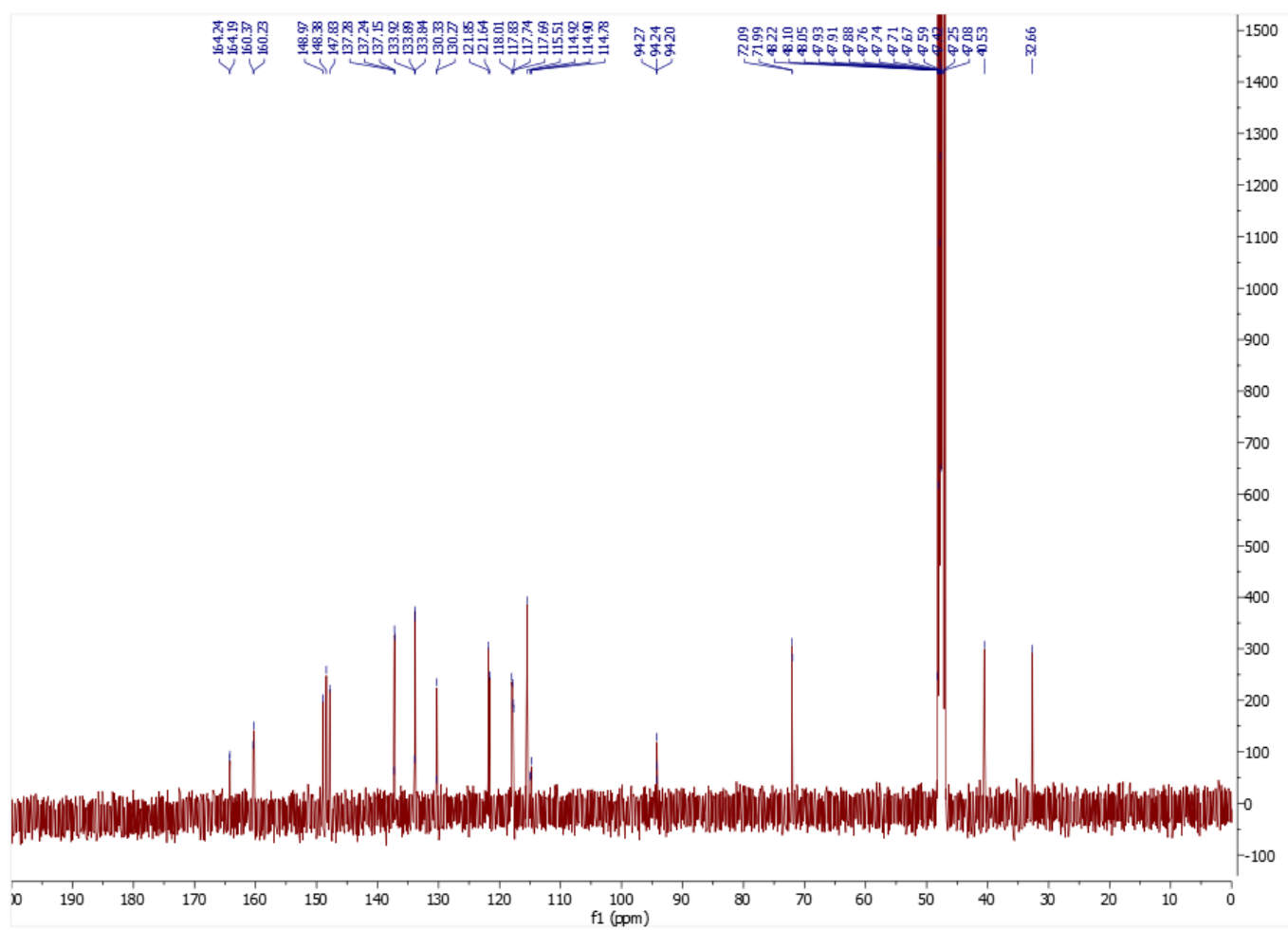


(8-Cyano-7-(methoxymethoxy)quinolin-2-yl)methyl (3,4-dihydroxyphenethyl)carbamate (MOM-CyHQ-N$D A)$.

${ }^{1} \mathrm{H}$ NMR $(500 \mathrm{MHz}$, chloroform- $d$ )

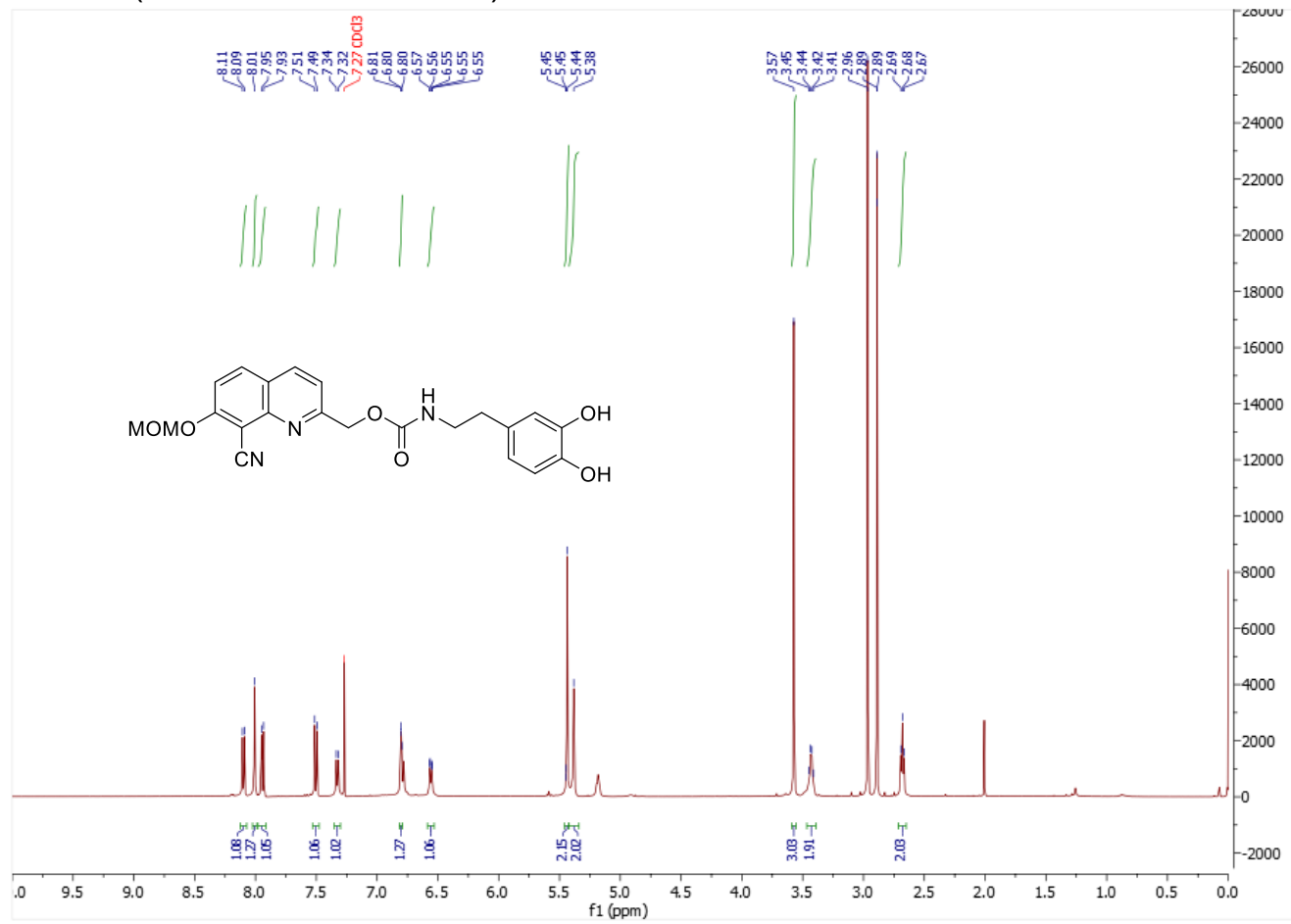

${ }^{13} \mathrm{C}$ NMR (126 MHz, chloroform- $d$ )

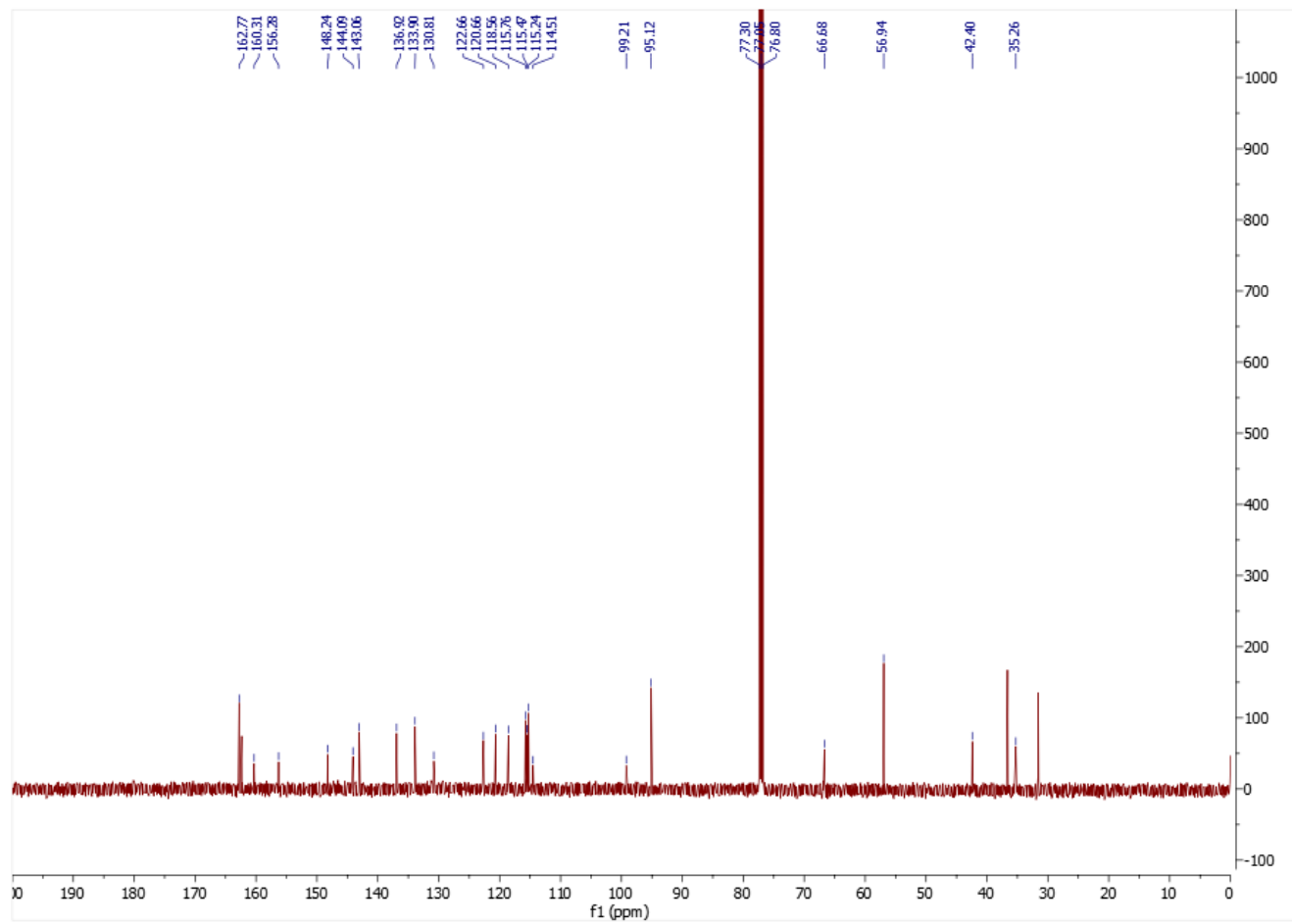


(8-Cyano-7-hydroxyquinolin-2-yl)methyl (3,4-dihydroxyphenethyl)carbamate (CyHQ-N-DA). ${ }^{1} \mathrm{H}$ NMR $\left(500 \mathrm{MHz}\right.$, methanol- $\left.d_{4}\right)$

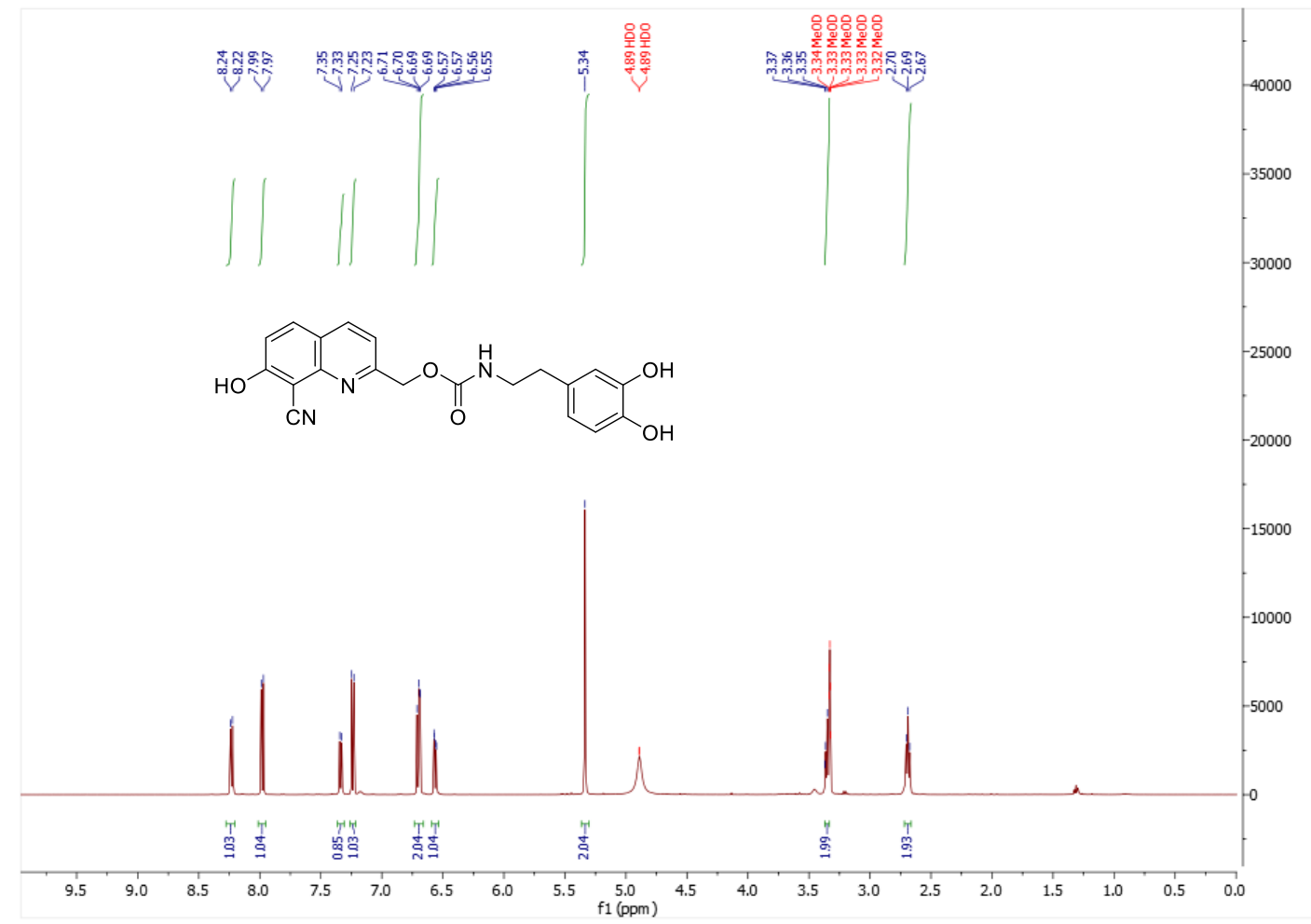

${ }^{13} \mathrm{C}$ NMR (126 MHz, methanol- $\left.d_{4}\right)$

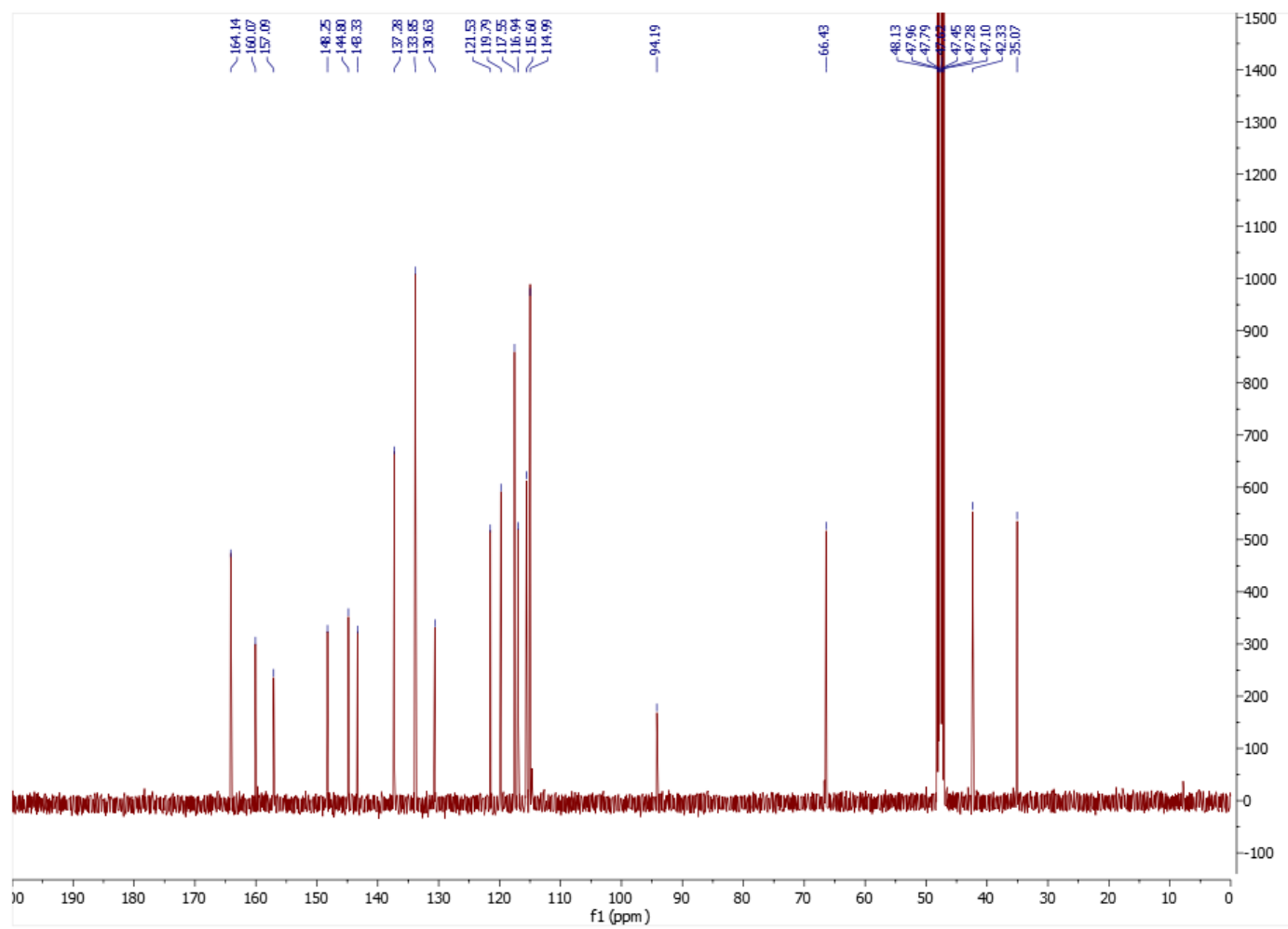


(8-Cyano-7-hydroxyquinolin-2-yl)methyl (3-((8-cyano-7-hydroxyquinolin-2-yl)methoxy)-3-

hydroxyphenethyl)carbamate and (8-cyano-7-hydroxyquinolin-2-yl)methyl (4-((8-cyano-7-hydroxyquinolin2-yl)methoxy)-3-hydroxyphenethyl)carbamate ((CyHQ) $2-\mathrm{N}, \mathrm{O}-\mathrm{DA})$

${ }^{1} \mathrm{H}$ NMR $\left(500 \mathrm{MHz}\right.$, methanol- $\left.d_{4}\right)$

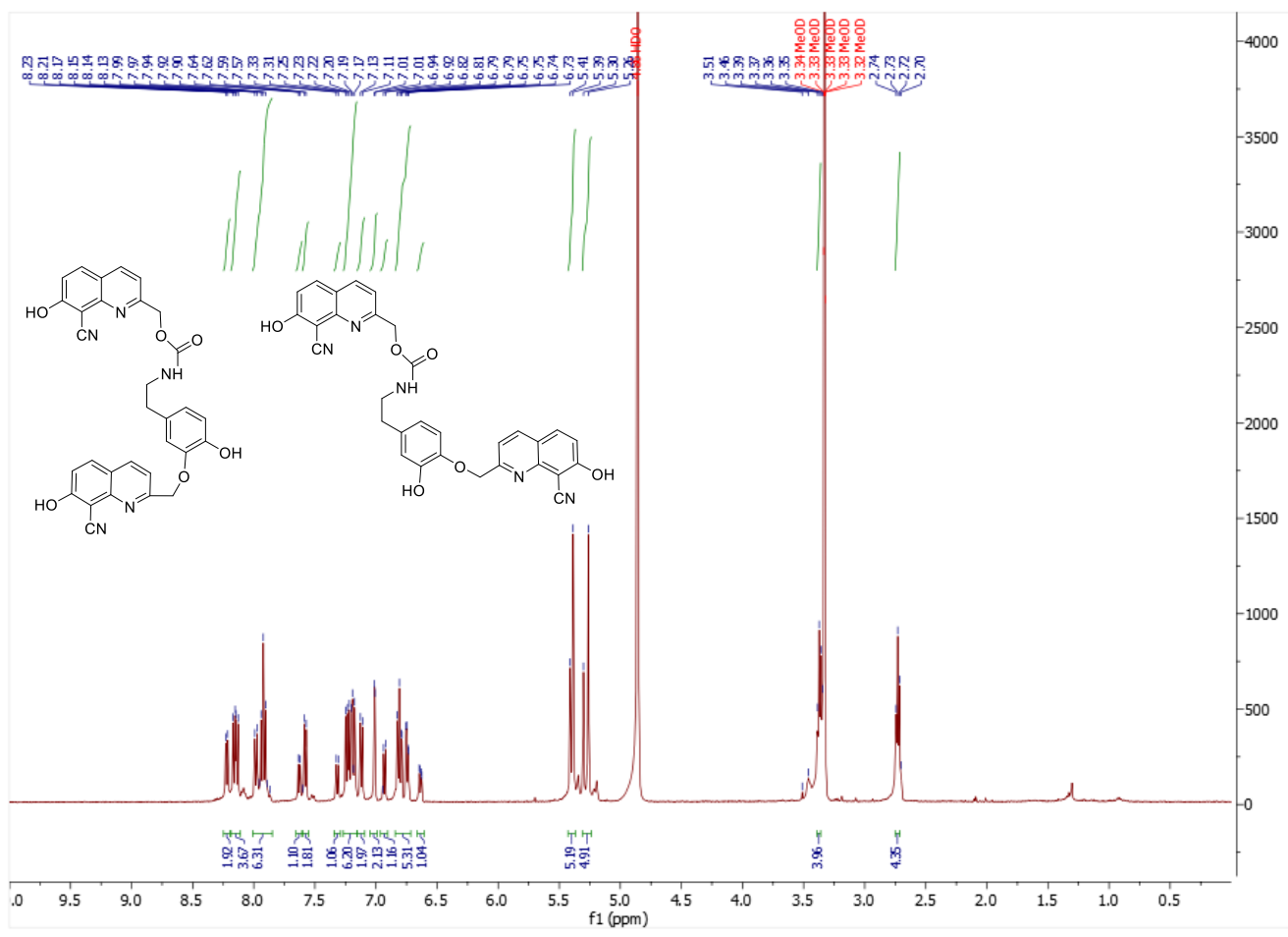

${ }^{13} \mathrm{C}$ NMR $\left(126 \mathrm{MHz}\right.$, methanol- $\left.d_{4}\right)$

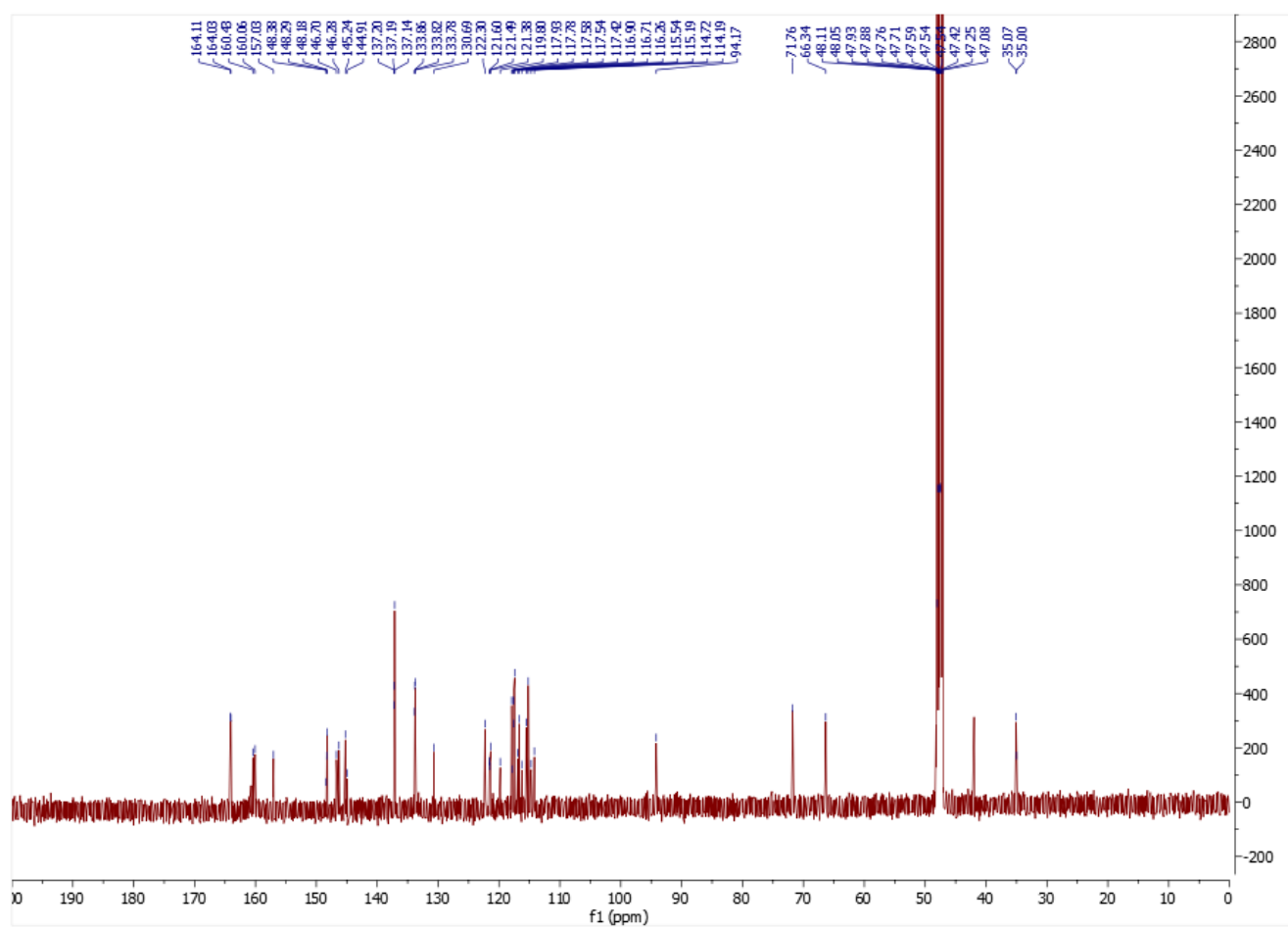


1-((8-cyano-7-hydroxyquinolin-2-yl)methyl)-1-ethyl-2-((2-methoxy-5-

sulfamoylbenzamido)methyl)pyrrolidin-1-ium (CyHQ-Sulpiride) 2,2,2-trifluoroacetate

Diastereomer 1

${ }^{1} \mathrm{H}$ NMR $\left(500 \mathrm{MHz}\right.$, methanol- $\left.d_{4}\right)$

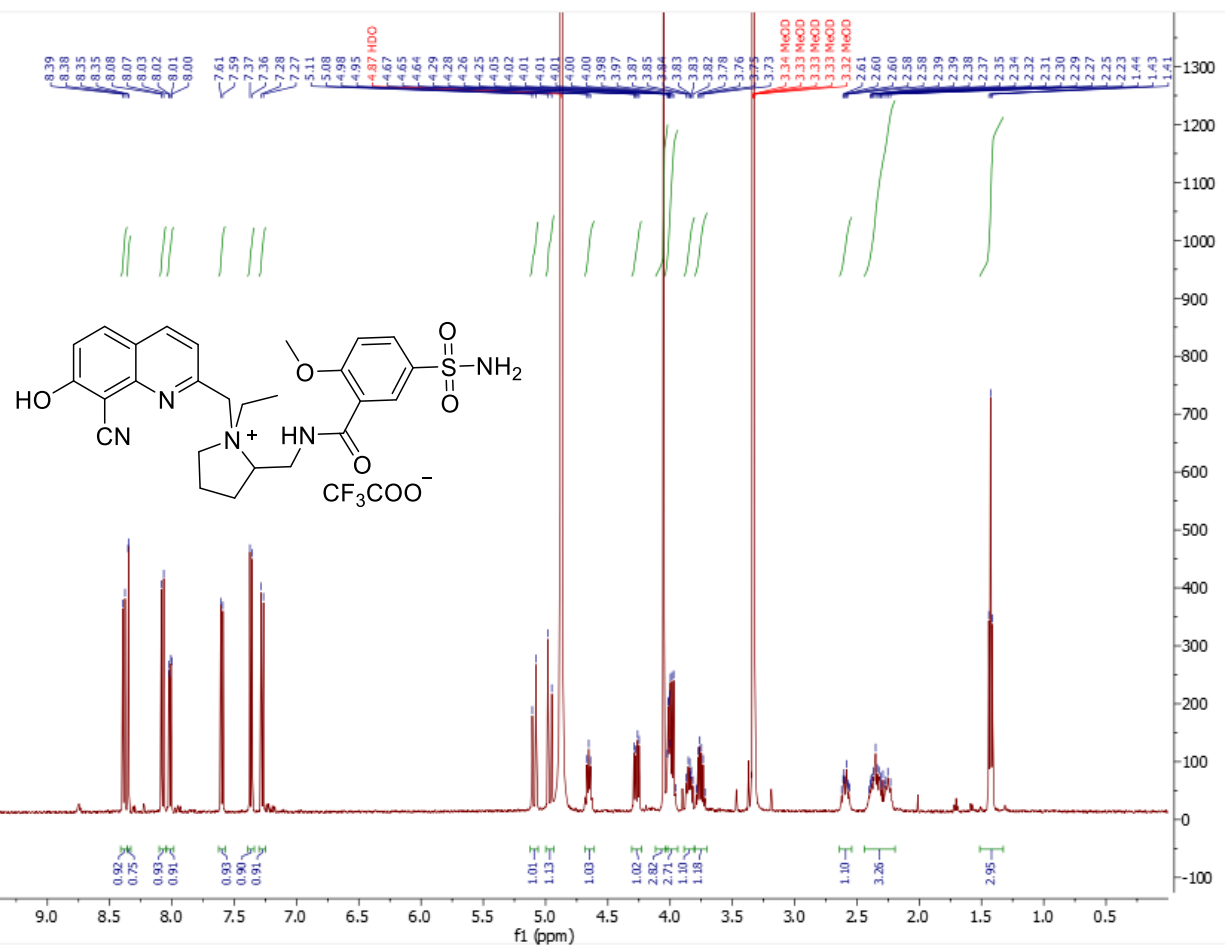

${ }^{13} \mathrm{C}$ NMR (126 MHz, methanol- $\left.d_{4}\right)$

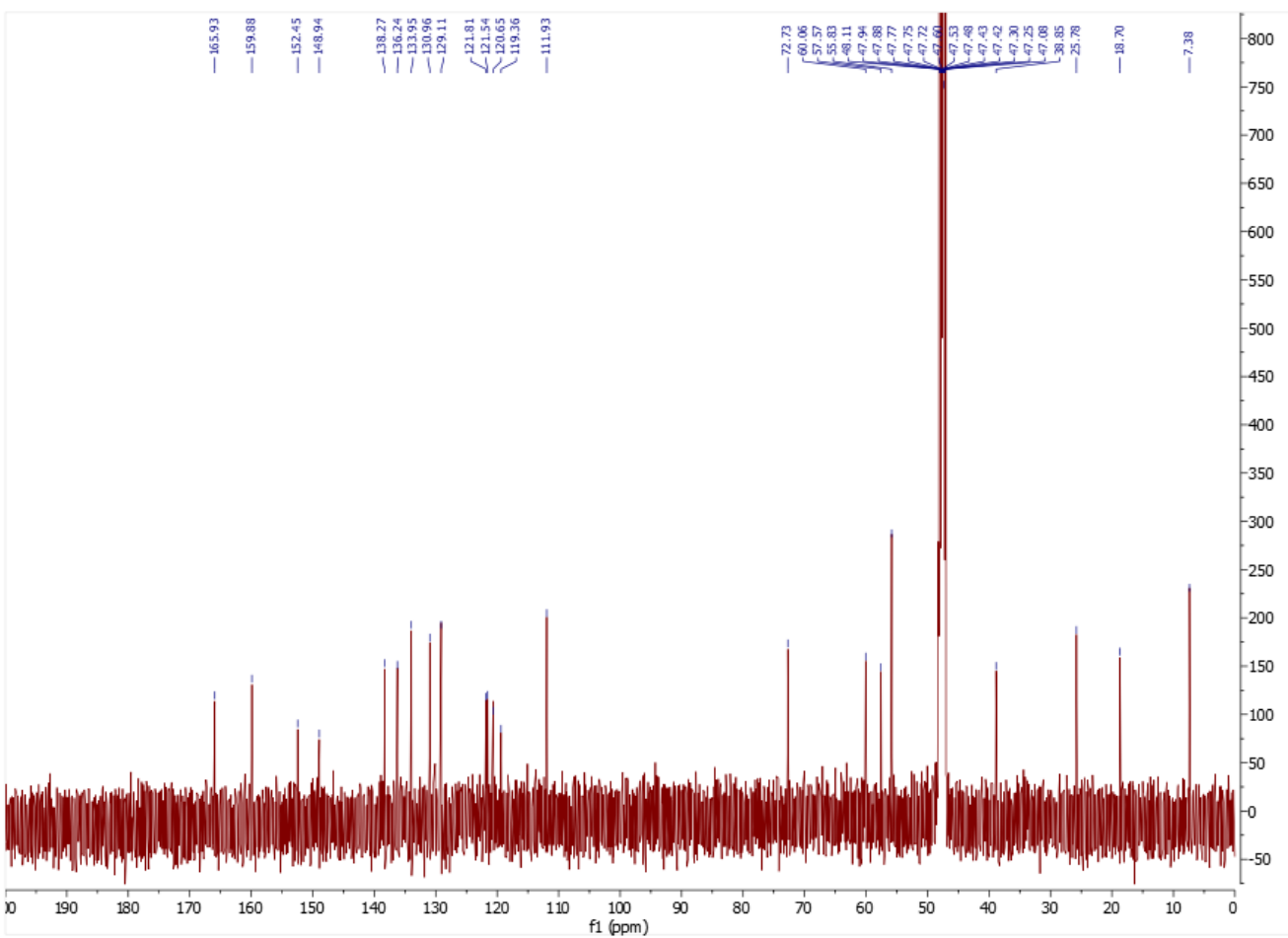


Mixture of Diastereomers 1 and 2

${ }^{1} \mathrm{H}$ NMR $\left(500 \mathrm{MHz}\right.$, methanol- $\left.d_{4}\right)$

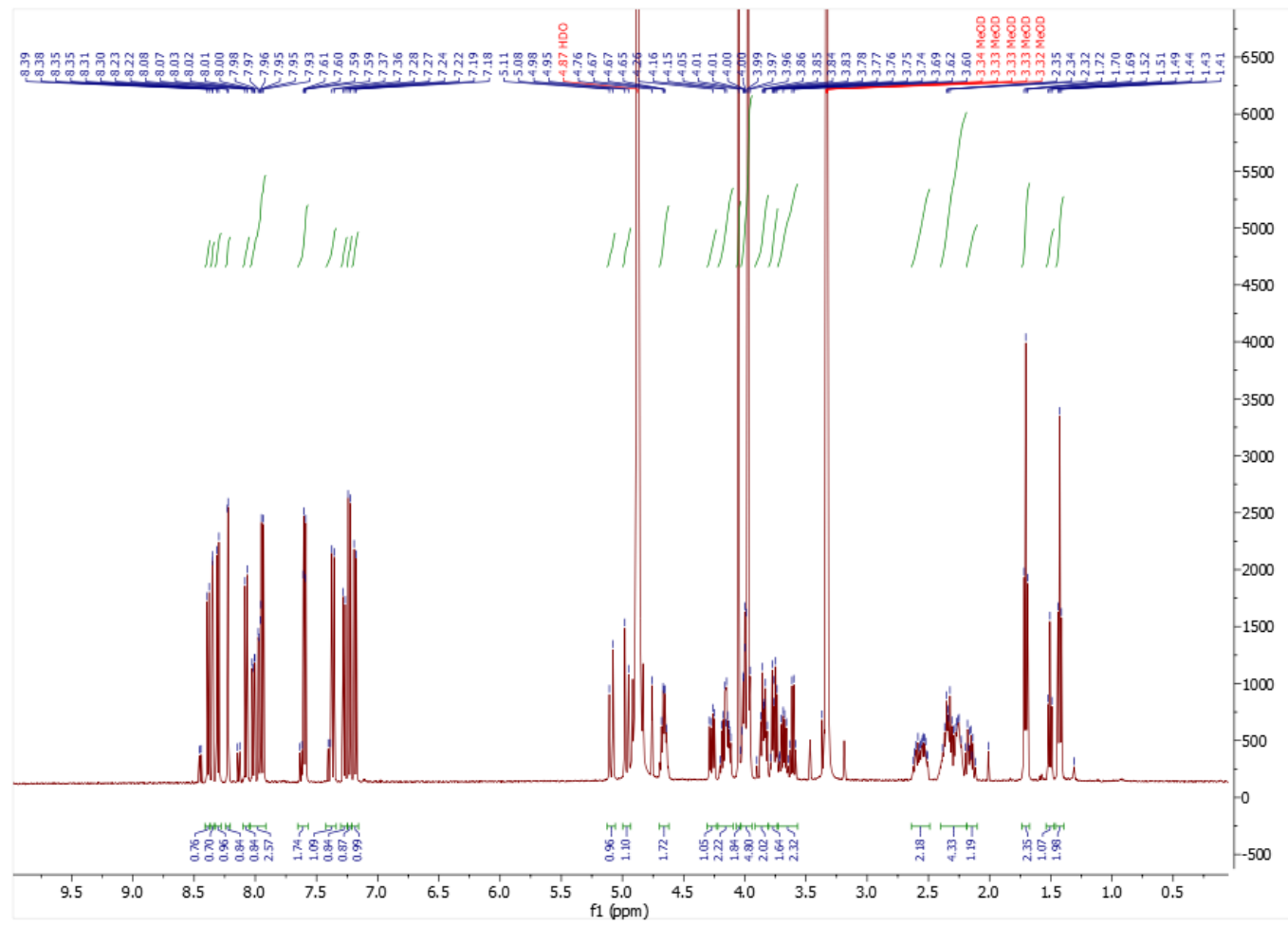

${ }^{13} \mathrm{C}$ NMR (126 MHz, methanol- $\left.d_{4}\right)$

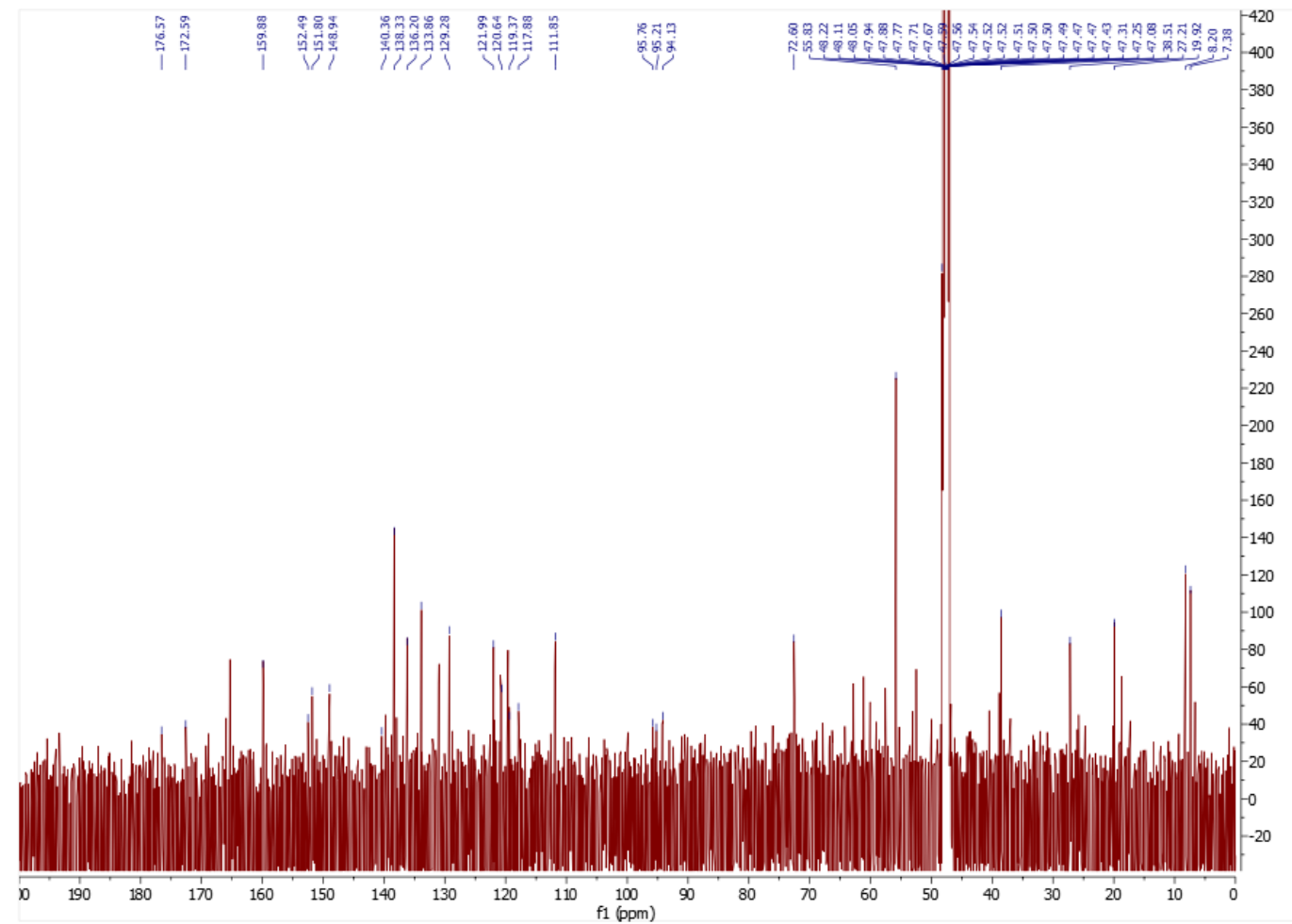




\section{References}

(1) Livingston, R. Behavior of Photochromic Systems. In Photochromism; Brown, G. H., Ed.; Wiley: New York, 1971, p 13-44.

(2) Adams, S. R.; Kao, J. P. Y.; Grynkiewicz, G.; Minta, A.; Tsien, R. Y. Biologically useful chelators that release $\mathrm{Ca}^{2+}$ upon illumination. J. Am. Chem. Soc. 1988, 110, 3212-3220.

(3) Furuta, T.; Wang, S. S. H.; Dantzker, J. L.; Dore, T. M.; Bybee, W. J.; Callaway, E. M.; Denk, W.; Tsien, R. Y. Brominated 7-hydroxycoumarin-4-ylmethyls: photolabile protecting groups with biologically useful cross-sections for two photon photolysis. Proc. Natl. Acad. Sci. U.S.A. 1999, 96, 1193-1200. DOI: 10.1073/pnas.96.4.1193

(4) Hennig, A.-L. K.; Deodato, D.; Asad, N.; Herbivo, C.; Dore, T. M. Two-Photon Excitable Photoremovable Protecting Groups Based on the Quinoline Scaffold for Use in Biology. J. Org. Chem. 2020, 85, 726-744. DOI: 10.1021/acs.joc.9b02780

(5) Hatchard, C. G.; Parker, C. A. A new sensitive chemical actinometer. II. Potassium ferrioxalate as a standard chemical actinometer. Proc. R. Soc. London, Ser. A 1956, 235, 518-536.

(6) Xu, C.; Webb, W. W. Measurement of two-photon excitation cross sections of molecular fluorophores with data from 690 to $1050 \mathrm{~nm}$. J. Opt. Soc. Am. B 1996, 13, 481-491.

(7) Xu, C.; Guild, J.; Webb, W. W.; Denk, W. Determination of absolute two-photon excitation cross sections by in situ second-order autocorrelation. Optics Lett. 1995, 20, 2372-2374.

(8) Albota, M. A.; Xu, C.; Webb, W. W. Two-photon fluorescence excitation cross sections of biomolecular probes from 690 to $960 \mathrm{~nm}$. Appl. Opt. 1998, 37, 7352-7356. 